\title{
Monitoring of a Reinforced Concrete Wharf Using Structural Health Monitoring System and Material Testing
}

\author{
Yann Lecieux ${ }^{1, * \mathbb{D}}$, Emmanuel Rozière ${ }^{2}$, Virginie Gaillard ${ }^{3}$, Cyril Lupi $^{1}$, Dominique Leduc ${ }^{1}$, \\ Johann Priou ${ }^{1}$, Romain Guyard ${ }^{1}$, Mathilde Chevreuil ${ }^{1}\left[{ }^{1}\right.$ and Franck Schoefs ${ }^{1}$ (i) \\ 1 Laboratoire GeM (UMR CNRS 6183), Université de Nantes, CNRS, École Centrale de Nantes, 2 , \\ rue de la Houssinière, 44322 Nantes CEDEX 3, France; cyril.lupi@univ-nantes.fr (C.L.); \\ dominique.leduc@univ-nantes.fr (D.L.); johann.priou@etu.univ-nantes.fr (J.P.); \\ romain.guyard@mines-albi.fr (R.G.); mathilde.chevreuil@univ-nantes.fr (M.C.); \\ franck.schoefs@univ-nantes.fr (F.S.) \\ 2 Laboratoire GeM (UMR CNRS 6183), École Centrale Nantes, CNRS, Université de Nantes, 1 Rue de la Noë, \\ 44321 Nantes CEDEX 3, France; emmanuel.roziere@ec-nantes.fr \\ 3 Laboratoire GeM (UMR CNRS 6183), ICAM, Département Génie Electrique, 35 Avenue du champ de \\ Manœuvres, 44470 Carquefou, France; virginie.gaillard@icam.fr \\ * Correspondence: yann.lecieux@univ-nantes.fr; Tel.: +332-5112-5550
}

Received: 25 February 2019; Accepted: 21 March 2019; Published: 27 March 2019

\begin{abstract}
This paper presents the Structural Health Monitoring (SHM) system developed for a port wharf of a freight terminal, in Saint-Nazaire, France. This concrete structure has been equipped with a multi-sensor system for the monitoring of concrete ageing. The measurement chain is designed to detect the penetration of chloride ions in order to quantify the risk of reinforcement bars corrosion. Modifications of the mechanical behavior of the structural elements of the wharf are also monitored. At first, the sensors embedded within the structure and the acquisition devices are described. The data from the monitoring performed during the first months of the structure service life are then presented. The concrete monitoring at early age providing data like temperature history, strain and resistivity is useful both for the wharf owner and the construction company since it indicates where concrete shrinkage is likely to cause cracking and gives an indicator of material hardening. These data were compared to the results of material tests carried out on concrete. The study shows that a measurement chain dedicated to the SHM could be a useful tool for validating the quality of the construction of a reinforced concrete structure before being used in the framework of long-term monitoring.
\end{abstract}

Keywords: SHM; Multi-sensors; DC electrical resistivity; optical fiber sensors; concrete; coastal structure

\section{Introduction}

Concrete harbor infrastructures are designed to perform their functions for a long period of service (100 years for the studied structure) while they are exposed to severe conditions (environment and loading) and they are often very sensitive to deterioration. Their design and maintenance strategy must be optimal in a highly competitive market and in the sense of a life-cycle management approach [1,2]. The monitoring of these structures can be of added value when consequences of the failure are severe, but also when the building method is innovative or when the maintenance is complex and costly. Within EC COST Action $1402^{1}$ a review of published papers and reports concerning monitoring

1 https:/ / www.cost-tu1402.eu (November 2014-April 2019). 
of wharves was carried out. It embraces only 15 structures in the world and less than 30 papers. The monitoring was largely devoted to the control of behavior (forces measurement in tie-rods or strain/displacement measurement performed on wall). More rarely, its objective is to improve structural models (less than half) or the identification of their parameters (less than $30 \%$ ). All the papers dealing with material properties are focusing on soil structure interaction $[3,4]$ or partially with the effect of environment on concrete properties [5].

The multi-sensor instrumentation presented here falls within the framework of the project iMARECO2 (french acronym for Monitoring for Maintenance, Reassessment and Optimal Design). It is a project approved by the Sea Innovation Cluster and managed by Keops Automation. The other partners are Université de Nantes, Nantes-Saint Nazaire Port and Bouygues Construction. It relies on the monitoring of the "Grand Quai" (main wharf) during its construction commissioned by Nantes-Saint Nazaire Port (Nantes-Saint Nazaire harbor, France). This has been built as an extension of an existing wharf at Montoir de Bretagne on the Loire estuary. The benefits of this project are multiple. First of all, the instrumentation will be used to optimize maintenance procedures. The real-time monitoring is generally of interest for the owner. In the long term, he can expect substantial costs saving in maintenance and a lifespan extension of the structure using relevant data for its decision support tools [6]. Secondly, the use of measurements during the construction stage could help the construction company to improve its building procedures. For the owner, the structural monitoring at early age associated with materials testing is useful to assess the actual performance of the structure delivered and its initial state (and not those of the specifications). Last, the instrumentation contributes to experience feedback. It should be emphasized that on pile wharf is a rare and prototype structure placed in complex environment generating multiple zones of degradation which causes additional costs as compared to conventional structures. During the design stage, the additional costs are generated by the lack of knowledge of several effects (environmental for example) and because construction standards do not take into account combined pathologies. After construction, additional maintenance costs are related to the difficulty of inspection, the complexity of the pathologies and the lack of experience feedback. In this context, there is a real need for experience feedback to optimize the operating costs including the cost of the measurement chain. Some studies [5,7-10] already contributed to this task but none took into account the benefit of the measurement performed at early age and during the first months of service life as it is done here in the framework of concrete coastal structures. The article presents the data provided by the measurement chain to monitor the structure at early age, evaluate its performance and monitor the evolution of concrete. Some sensors installed on the structure to detect long-term disorders are also sensitive to changes in the concrete occurring during the first months. These data are discussed and compared with the results of tests carried out on concrete in laboratory which allows to identify the most relevant methods and data for manufacturing monitoring.

In the first part of the article, the structure, the sensors (technology and positioning), and concrete testing methods are described. The second part presents the methods used to analyze the data from structural monitoring and material testing. Finally, the results are compared and discussed to identify useful data during the monitoring of the manufacturing process.

\section{Materials and Methods}

\subsection{The Structure}

The wharf is a $350 \mathrm{~m}$ long, $50 \mathrm{~m}$ wide on pile structure. It is composed of partly precast reinforced concrete elements. Precast parts are supported by a mesh of 579 steel piles distributed over 10 longitudinal rows and 56 transverse rows. Depending on the areas of the wharf, different service loads for parcel storage (offshore wind turbine nacelles) are planned $\left(6,10\right.$ or $15 \mathrm{ton} / \mathrm{m}^{2}$ ) as well as crane tracks. The reinforced concrete platform is covered with a layer of embankment and asphalt. The platform is also equipped with bollards and berths. To stabilize the banks on which the platform is built, concrete inclusions and a system comprising a double sheet pile wall and tie rods have been 
installed (see Figure 1). The construction took over two years for a cost of around 40 million Euros and was achieved at the end of 2017.

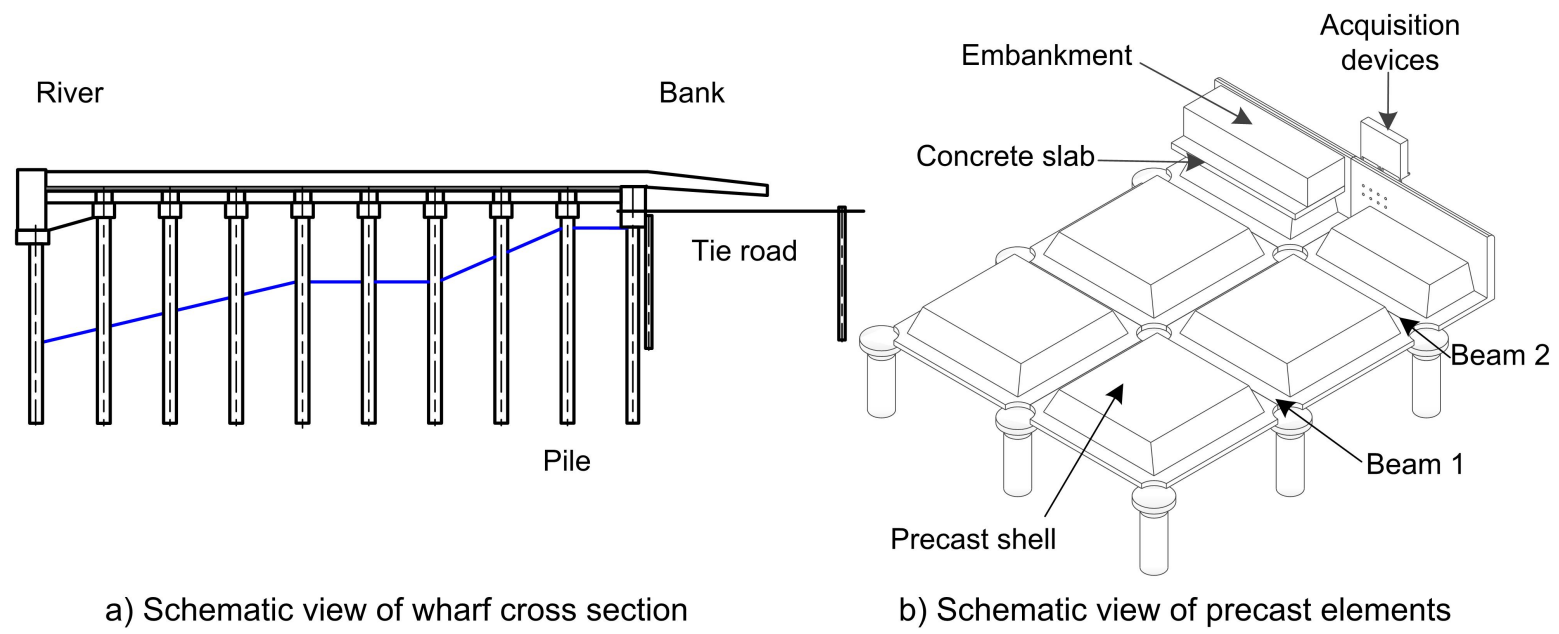

Figure 1. Schematic view of the wharf.

The precast elements are concrete shells. They are embedded in the heads of steel piles (see Figures 1 and 2).

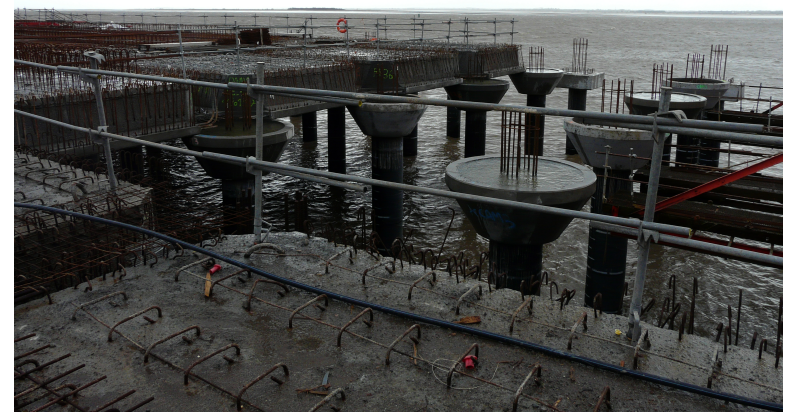

Figure 2. The wharf during construction (precast shell elements and metal piles).

The structural elements of the wharf called "beams" are delimited by the volumes between the prefabricated shells. Reinforcements are installed in this volume and then it is filled with concrete during the construction phase. Two structural elements located at the end of the platform are equipped with sensors (see Figures $1 \mathrm{~b}$ and 3). 


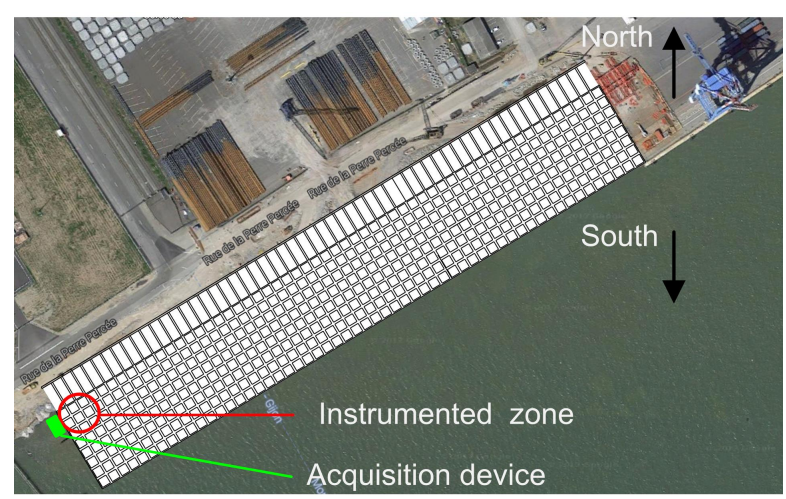

Figure 3. Zone of interest.

\subsection{Technology and Positioning of the Sensors}

The two beams in the instrumented zone are equipped with a multi-sensor system for monitoring chloride ion penetration and ageing according to the drawings in Figure 4.

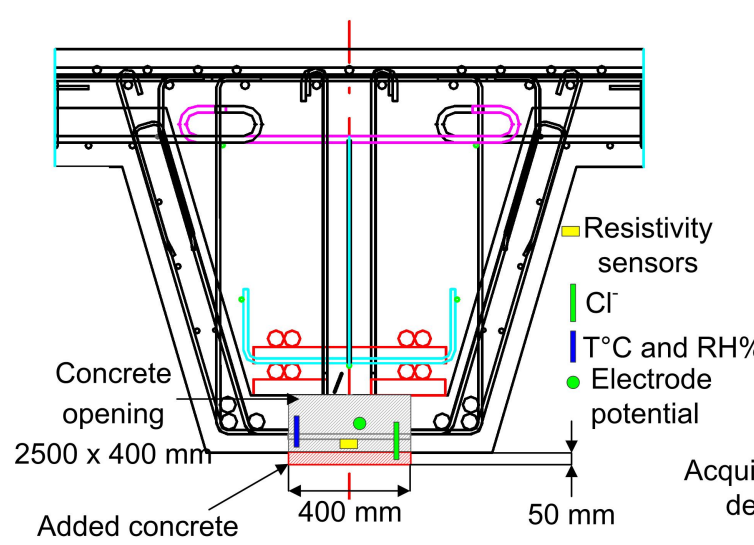

a) Beam cross sectional view

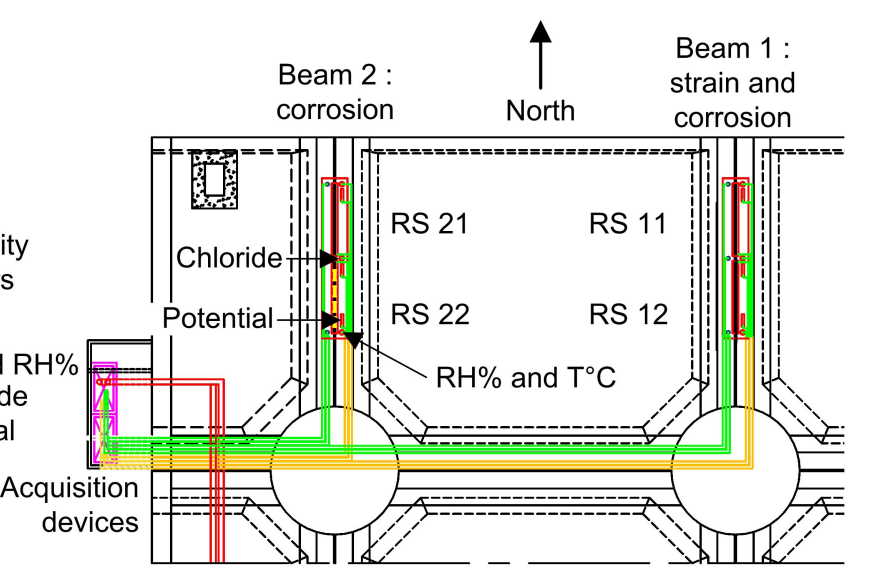

b) Top view

Figure 4. Location of potential, chloride and resistivity sensors (referenced RS i).

Each beam is equipped with:

- 2 resistivity sensors of the same technology as those used in [11];

- 3 chloride sensors (physicochemical sensors with 3 electrolyte levels);

- 3 sensors for the measurement of Direct Current (DC) electrical potential of reinforcement bars;

- 3 combined humidity and temperature probes;

- 6 Temperature probes Pt 100 and 14 thermocouples;

- 2 optical fibers for simultaneous measurement of strain and temperature with a Brillouin-Rayleigh optical fiber interrogator.

These sensors are selected to measure changes in concrete related to the aging of the structure. Electrical DC resistivity measurement aims to detect the penetration of chloride ions into the concrete cover [12-15]. The resistivity depends on the porosity of the concrete, the water content, the concentration of chloride ions in the electrolyte and the temperature of the medium. Resistivity sensors are therefore used in combination with humidity and temperature sensors. Chloride sensors are designed to detect the front of chloride ion when it first reaches the reinforcement. The objective is to detect the presence of chloride before the corrosion process begins. DC electrical potential measurement is then used to predict the initiation of corrosion, before cracking appears. Finally, optical fibers are used to localize concrete cracking due to thermal changes or corrosion. 
This instrumentation is deliberately redundant in order to be able to compare the performance of the sensor technologies in the long term.

The Pt 100 probes and thermocouple sensors have been calibrated in temperature chambers. Humidity sensors were placed in temperature chambers in sealed containers containing saturated saline solutions according to standard [16]. The resistivity sensors were calibrated in a homogeneous medium including the actual reinforcement bars (see Figure 5) as described in Section 3.2.

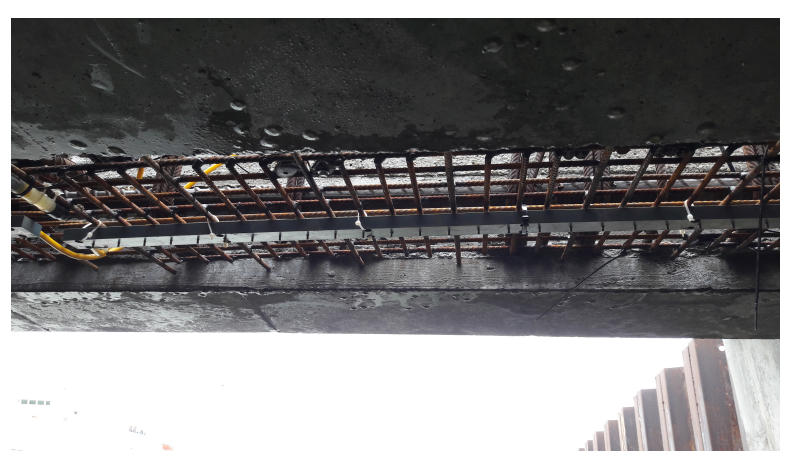

Figure 5. View of reinforcement bars and resistivity sensors for on-site measurement (bottom view).

Beam 1 (see Figure 4) is also equipped with 15 optical fiber extensometers (see Figure 6). Extensometers are positioned according to the symmetry plane of the beam at different distances of the neutral fiber. The objective of the strain measurements is to understand the actual behavior of the structural element. Because of its proportions, its behavior no longer falls within the validity domain of beam theory. Strain gauges are combined with strain-free Bragg gratings to measure temperature. The entire chain is calibrated by performing heating and cooling cycles in a thermal chamber.

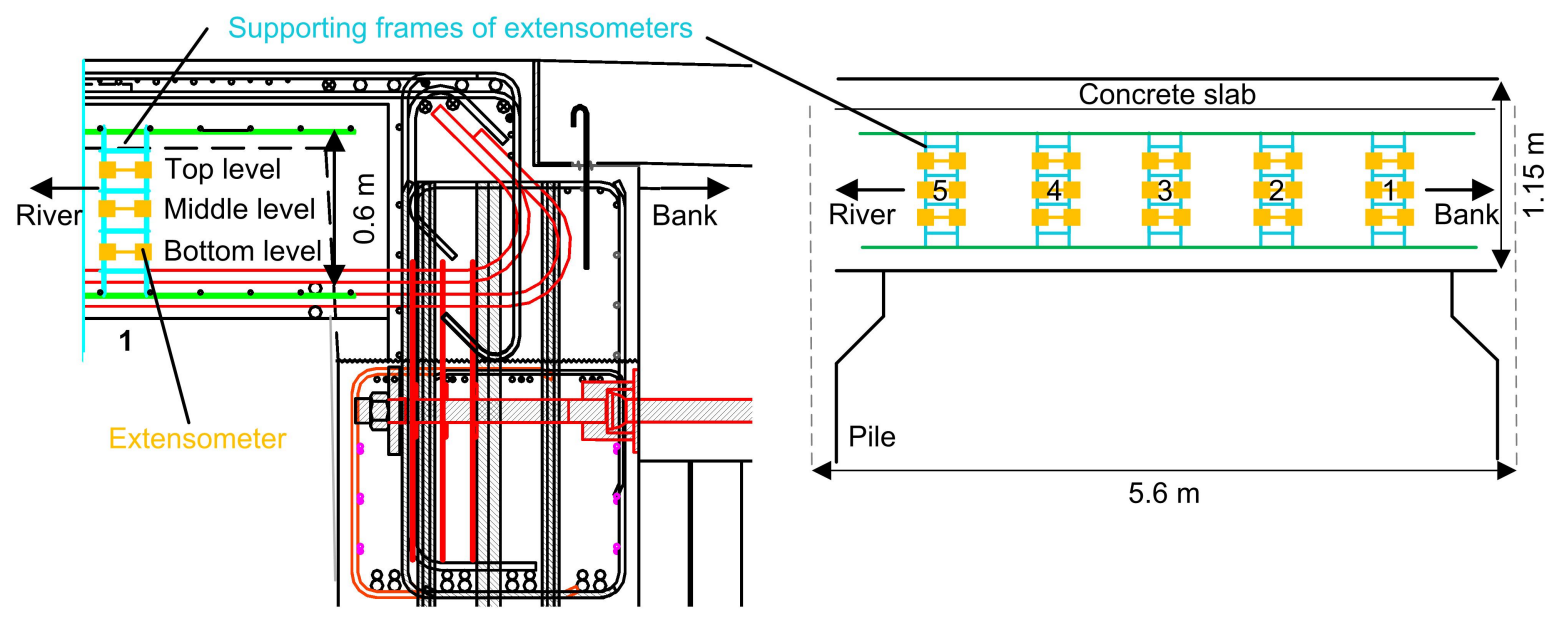

a) Beam sectional view (Plan of the tie road)

b) Schematic view of extensometers positioning

Figure 6. Instrumentation for strain measurement (beam 1).

Thermocouple or $\mathrm{Pt} 100$ temperature sensors are positioned near resistivity sensors and strain gauges. They are used for the compensation of thermal effects on strain and resistivity measurements. Thermocouples, used in large numbers due to their low cost, are used to measure thermal gradients. The sensors whose data are analyzed in the following are marked on Figure 7. 


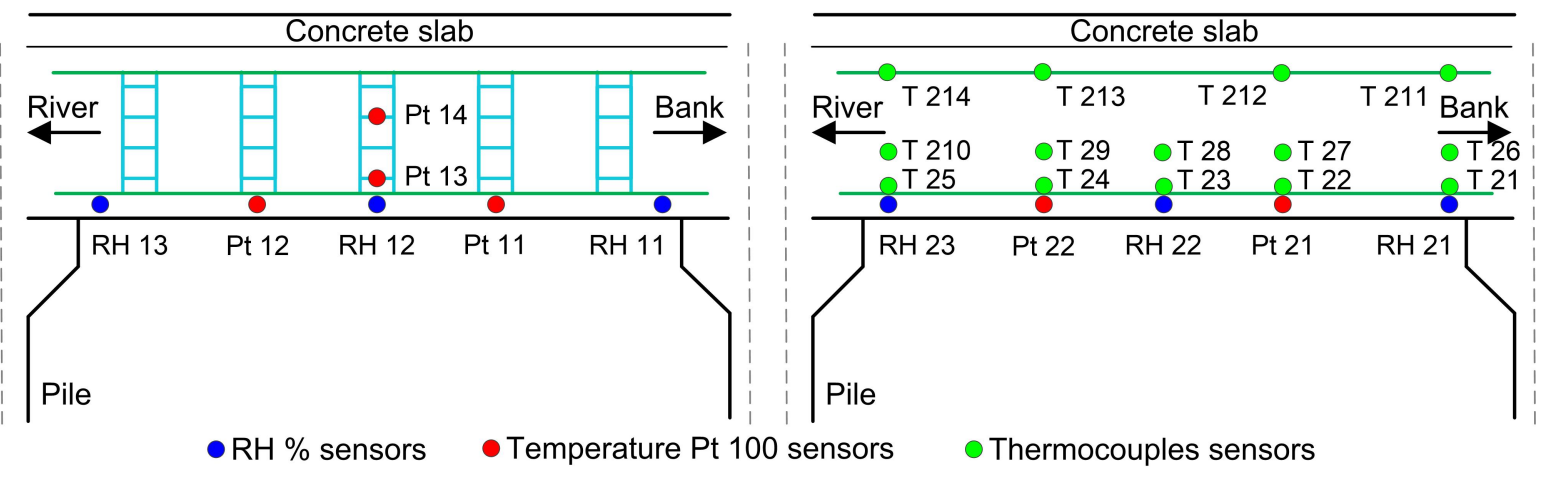

a) Schematic view of beam 1

b) Schematic view of beam 2

Figure 7. Temperature and humidity sensors in beam 1 and beam 2.

The references of the sensors used and the names of the suppliers are listed in Table A1 in the Appendix A.

\subsection{Acquisition Device}

Data acquisition for chloride, temperature, potential and humidity sensors is carried out with a NI cDAQ-9133 acquisition unit which includes several acquisition cards. The central acquisition unit records:

- Voltages from chloride sensors with an accuracy (for the acquisition device only) of $10 \mathrm{nV}$,

- Thermocouple data: after thermocouple calibration and cold junction compensation, the maximum temperature uncertainty given by the thermocouples over the measuring range $\left[-20^{\circ} \mathrm{C} ; 40^{\circ} \mathrm{C}\right]$ is $0.9{ }^{\circ} \mathrm{C}$ (defined as maximum to minimum deviation).

- Voltages from temperature and humidity sensors with a maximum temperature uncertainty over the measuring range $\left[-20^{\circ} \mathrm{C} ; 40^{\circ} \mathrm{C}\right]$ of $0.25^{\circ} \mathrm{C}$. The humidity measurement uncertainty over the same range is $1 \%$.

- Data from platinum resistance thermometers of type Pt 100 whose maximum temperature uncertainty over the measuring range $\left[-20^{\circ} \mathrm{C} ; 40^{\circ} \mathrm{C}\right]$ is $0.2^{\circ} \mathrm{C}$.

The acquisition unit requires a $24 \mathrm{~V}$ DC power supply. It has been connected to an autonomous circuit. The $24 \mathrm{~V}$ DC came from batteries regularly replaced and connected to the acquisition unit via a converter, ensuring stable DC voltage during the initial connection of the installation. In a second stage, photovoltaic panels of about $0.5 \mathrm{~m}^{2}$ were installed, in order to provide a maximum power of about $100 \mathrm{~W}$.

The acquisition unit has an embedded operating system. It has a 2 GB memory and offers the possibility to connect an SD card. A Labview-coded program was developed in laboratory to record data from all the acquisition cards simultaneously at a desired frequency. The data is recorded on an SD card connected to the control unit. The acquisition of the signals of the Bragg grating optical fiber sensors (FBG) during the first months is carried out punctually with a portable device (HBM FS42 Portable BraggMETER). The resistivity measurement is carried out punctually with a resistivimeter ABEM terrameter LS, which is used as standard by geophysicists and is suitable for civil engineering applications [11,17].

The whole installation (batteries, electronics, central unit, etc.) allowing data acquisition has been integrated into waterproof boxes to protect it from external aggressions (wind, rain, tide, etc.). The boxes are positioned at the end of the platform as shown in Figures 3 and 4. 


\subsection{Construction Program}

The installation of the measuring chain was carried out in conjunction with the progress of the construction works according to the following program:

- $t_{0}-3$ days, the precast concrete shells are positioned on the pile heads;

- $t_{0}-3$ days $<t<t_{0}$, the reinforcement bars are placed in the lower part of the precast concrete shell in parallel with the installation of the sensors dedicated to corrosion measurement;

- $t=t_{0}$, the concrete is poured in the lower area (zone of resistivity sensors). Data acquisition is started at time $t_{0}$;

- $t_{0}<t<t_{0}+2$ days, the reinforcement bars are placed in the beams in parallel with the installation of the strain measurement sensors;

- $t=t_{0}+9$ days, the concrete is poured at the junction of the concrete shells to form the beams;

- $t=t_{0}+16$ days, the concrete slab is poured over the concrete shell.

The different areas listed above are shown in Figure 8 and limited by dashed red lines.

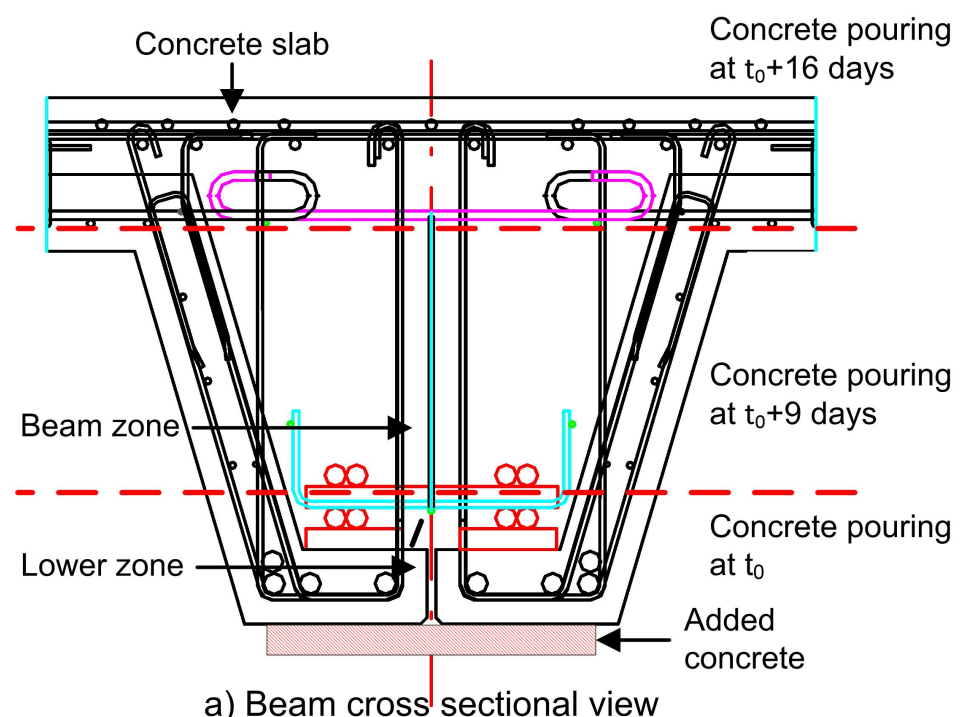

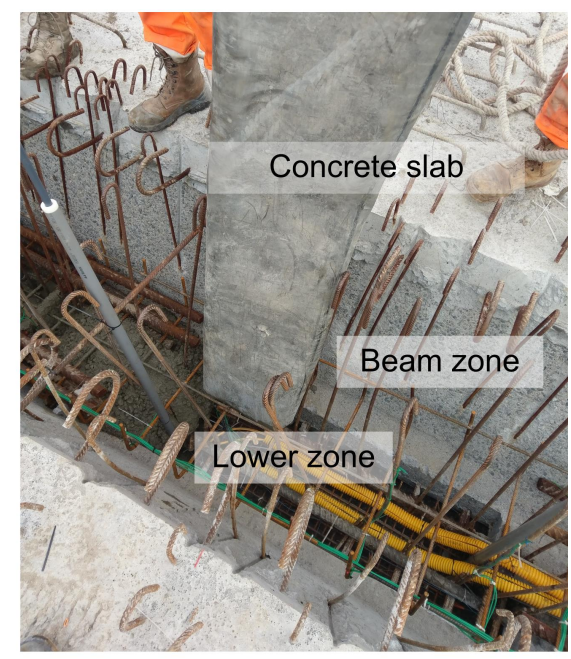

b) On site view

Figure 8. Identification of zones of the structure cast at different times.

\subsection{Materials and Mixture Used on Site}

The composition of cast-in-place concrete is given in Table 1 . The strength class was C40/50 and the mixture complied with the requirements of French Standard NF EN 206-1 for XA2 exposure class; i.e., minimum binder content of $350 \mathrm{~kg} / \mathrm{m}^{3}$ and maximum Effective water to Binder (Weff/Beq) ratio of 0.50. Portland cement CEM I 52.5 N SR3 was used to provide sulfate resistance. The actual Weff/Beq ratio was 0.45 .

Table 1. Composition of concrete mix.

\begin{tabular}{cc}
\hline Component & Content $\left(\mathbf{k g} / \mathbf{m}^{3}\right)$ \\
\hline Gravel 11/22 & 740 \\
Gravel 2/10 & 300 \\
Sand 0/4 & 810 \\
Cement CEM I 52.5 N SR3 (C) & 360 \\
Plasticizer & 3.8 \\
Water effective & 161 \\
\hline
\end{tabular}




\subsection{Material Tests Performed in Laboratory}

In parallel with in-situ measurements, material tests were performed in controlled laboratory conditions in order to characterize the concrete used in the beams. On site, during the casting of the beams, 40 cylindrical samples of $110 \mathrm{~mm}$ diameter and $220 \mathrm{~mm}$ height, and one concrete prism of $70 \times 70 \times 280 \mathrm{~mm}^{3}$ were also made with the same concrete. After one day, they were stored in water at 3 different temperatures: 4 were stored at $10{ }^{\circ} \mathrm{C}, 4$ at $40{ }^{\circ} \mathrm{C}$ and the other at $20^{\circ} \mathrm{C}$ in order to perform maturity analysis and to determine activation energy. These samples were used to perform the following measurements:

- $\quad$ Porosity accessible to water;

- Compressive strength, (Maturity method [18]);

- $\quad$ Elastic modulus.

Moreover, the same day, a piece of the concrete poured in the beam was taken and brought to the laboratory in less than one hour, in order to cast a $7 \times 7 \times 28 \mathrm{~cm}^{3}$ specimen used to measure the autogenous deformation [19].

\subsubsection{Porosity}

An essential characteristic of reinforced concrete is its porosity since the pore structure has a strong influence on the diffusivity of chloride ions in concrete. It is therefore a determining factor in the durability of concrete [20]. After 90-day wet curing, water porosity $\mathrm{P}(\%)$ was assessed on three concrete discs by the vacuum water saturation method, according to French standard NF P18-459 [21]. It is then deduced from mass measurements and Equation (1):

$$
P=\frac{M_{\mathrm{sat}}-M_{d}}{M_{\mathrm{sat}}-M_{w}}
$$

where $M_{\text {sat }}$ is the mass of saturated sample in air, $M_{d}$ is the mass of oven-dried sample and $M_{w}$ is the mass of saturated sample in water.

\subsubsection{Compressive Strength and Elastic Modulus}

The compressive strength was assessed after 1, 2, 7, and 28 days on the specimens stored in water at different temperatures. Three specimens for each storage temperature were tested at each age. A non-destructive method based on the frequency of vibration was used to monitor the evolution of elastic properties on the set of specimens stored at $20^{\circ} \mathrm{C}$. The natural frequencies of the concrete cylinders were measured with the Grindosonic equipment. The elastic modulus $\mathrm{E}$ was deduced by using the Spinner and Tefft model [22].

\subsubsection{Autogenous Deformation}

Autogenous deformation has been defined as the bulk deformation of a closed, isothermal, cementitious material system not subjected to external forces [23]. It mainly results from the hydration of cement. As studied concrete is located in the central part of the beam and in a relatively wet environment, drying is not expected to have a significant influence (at least during the presented study). Thus the laboratory monitoring consisted in measuring the length variations of $7 \times 7 \times 28 \mathrm{~cm}^{3}$ specimen in sealed conditions. The sample was casted in laboratory with concrete taken on the construction site one hour before. After 24 -h sealed curing at $20^{\circ} \mathrm{C}$, the specimen was removed from formwork and immediately covered with a double-layer of adhesive aluminum tape. The autogenous deformation was monitored during one year. The mass-loss did not exceed $0.2 \%$ of initial mass. 


\section{Post-Treatment of Data for Comparison and Analysis}

\subsection{Analysis of Strain Measurement Performed with Fiber Bragg Gratings}

Extensometer with Bragg grating optical fiber provides a Bragg wavelength $\lambda_{0}$. The offset $\Delta \lambda$ with respect to a reference state is linked to thermal and mechanical strain. According to Equation (2):

$$
\frac{\Delta \lambda}{\lambda_{0}}=\left(1-\frac{n_{\mathrm{eff}}^{2}}{2}\left[p_{12}-v_{f}\left(p_{11}+p_{12}\right)\right]\right) \varepsilon+\xi \Delta t
$$

where $\Delta t$ is temperature change, $\varepsilon$ is the axial strain of the FBG, $n_{\text {eff }}$ is the effective index of the mode propagating in the core of the fiber, $v_{f}$ is the Poisson coefficient of the fiber, $p_{11}$ and $p_{12}$ are photo-elastic coefficients while $\xi$ is the thermo-optic coefficient. Equation (2) can also be written in a condensed form:

$$
\frac{\Delta \lambda}{\lambda_{0}}=K \varepsilon+\xi \Delta t
$$

The value of $\mathrm{K}$ is given by the sensor supplier in the calibration report. According to Ferdinand [24], $\xi=\frac{1}{n_{\mathrm{eff}}} \frac{\mathrm{d} n_{\mathrm{eff}}}{\mathrm{d} T} \simeq 7 \times 10^{-6} \mathrm{~K}^{-1}$. It has been evaluated by thermal calibration for each sensor. In addition to the strain measurements, strain-free FBGs are positioned between top and middle levels (see Figure 6) to ensure thermal compensation of the system. During the first days, the relevant information that can be calculated using these extensometers is the shrinkage of the concrete at the core of the structure. It is calculated from the Equation (4) knowing the thermal dilatation coefficient of the concrete $\alpha_{\text {concrete }}$ (with $\alpha_{\text {concrete }}=10 \times 10^{-6} /{ }^{\circ} \mathrm{C}$ )

$$
\varepsilon=\alpha_{\text {concrete }} \Delta T+\varepsilon_{\text {shrinkage }}
$$

\subsection{Analysis of Resistivity Measurement Performed with a Multi Electrodes Wenner Probe Embedded in Concrete}

Concrete resistivity is measured in this study by electrical tomography. The resistivity noted $\rho(\Omega . m)$, quantifies the propagation in a medium of an electric current $I$ (A) continuously injected by an electrode embedded in concrete (see Figure 9). The usual electrical prospecting devices generally consist of two injection electrodes (A and B) and two electrodes $\mathrm{M}$ and $\mathrm{N}$, allowing the measurement of the potential difference $\Delta V$. The apparent resistivity for a 4-electrodes system is computed as follows:

$$
\rho_{a}=G \frac{\Delta V}{I}
$$

$G\left(\mathrm{~m}^{-1}\right)$ is the geometric factor that can be analytically calculated for a semi-infinite medium. For a Wenner [25] device as shown in Figure 9 and used for concrete beam monitoring, the inter-electrode distance is $\mathrm{AM}=\mathrm{MN}=\mathrm{NB}=\mathrm{a}$ (with $\mathrm{a}=25 \mathrm{~mm}$ ). The sensor used on the wharf has 32 electrodes. Using a Wenner sequence of 4 electrodes [11,17], 129 different measurements can be carried out. Thanks to these measurements, a mapping of the apparent resistivities in a plane located under the sensor (called pseudo section of apparent resistivity) is obtained.

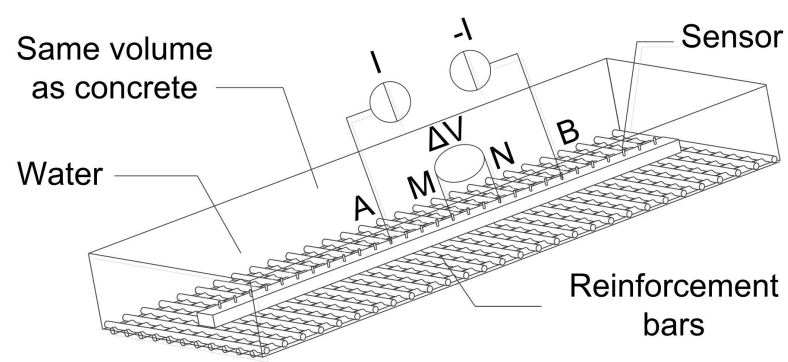

Figure 9. Principle of measurement and schematic view of the experimental set-up. 
When the medium is of finite dimension such as studied beams, the geometric factor cannot be analytically computed (contrary to what is done in Geophysics [26]). Indeed, the latter depends on the geometry of the concrete beam and the reinforcement. As proposed by Du Plooy and al. [17], a numerical assessment of $G$ was performed. The sensors have been calibrated in a homogeneous medium (here water of known resistivity) of the same volume as the concrete with reinforcement bars used on site (see Figures 5 and 9).

\subsection{Equivalent Age}

Significant temperature gradients are likely to be generated in the studied beam due to dimensions of cross section of the wharf structural elements [27]. Early-age thermal cracks result from either differential expansion within a concrete element during heating causing internal restraint or by external restraint due to contraction during cooling stage. The main parameters involved in early age thermal cracking, i.e., strength, modulus, shrinkage, and creep, depend on concrete temperature history. Maturity method [18] allows estimating the properties of concrete from the measured temperature evolution of in-place concrete and laboratory tests in controlled conditions. This method is based on the measurements performed at different times of the compressive strength of the samples stored at different temperature (see Section 2.6.2). First, the evolution of the compressive strength of the samples stored at $20^{\circ} \mathrm{C}$ is fitted with a function adapted from the mathematical expression given in Eurocode 2:

$$
f_{c}^{T_{\text {ref }}}=f_{\mathcal{C}_{28 \mathrm{~d}}} \mathrm{e}^{s\left(1-\sqrt{\frac{28}{t}}\right)}
$$

where $f_{c 28 \mathrm{~d}}$ is the compressive strength measured at 28 days, $T_{\text {ref }}=20^{\circ} \mathrm{C}$ is the reference temperature and $s$ is the adjustable parameter. In Eurocode 2 (NF EN 1992-1-1, 2005 [28]), the coefficient $s$ takes into account the type of cement, from rapid hardening cements to low heat cements:

- $\quad 0.20$ for cements CEM 42.5 R, CEM 52.5 N, and CEM 52.5 R (Class R);

- $\quad 0.25$ for cements CEM 32.5 R, CEM 42.5 N (Class N);

- $\quad 0.38$ for cements CEM $32.5 \mathrm{~N}$ (Class S).

In the paper the coefficient $\mathrm{s}$ was adjusted to fit the actual strength development of the studied concrete. The value $s=0.21$ was chosen to obtain the exact value of the strength measured at 2 days (see Table 2). The value is consistent with the intervals of Eurocode. The evolution of the compressive strength of the samples stored at $20^{\circ} \mathrm{C}$ serves as a reference. The underlying idea of the maturity method is to superimpose the evolutions of compressive strength with time at temperature $T$ to this reference with the help of an equivalent age:

$$
f_{c}^{T}(t)=f_{c}^{T_{\text {ref }}}\left(t_{e q}(T)\right)
$$

Equivalent age is the duration when concrete has to be stored at temperature in order to reach the same level of maturity as it would reach in the curing conditions defined by the temperature history $T(t)$. The influence of temperature on cement hydration and related properties can be taken into account through Arrhenius equation and activation energy $E_{a}$. This allows calculating the equivalent age $t_{e q}$ from the Equation (8), given by Freiesleben Hansen et Pedersen [29]:

$$
t_{e q}(T)=\int_{0}^{t} \mathrm{e}^{-\frac{E_{a}}{R}\left(\frac{1}{T(\tau)}-\frac{1}{T_{\text {ref }}}\right)} \mathrm{d} \tau
$$

where $t_{e q}$ is the equivalent age at reference temperature (days), $E_{a}$ is the apparent activation energy $[\mathrm{J} / \mathrm{mol}], R$ is the gas constant $8.314 \mathrm{~J} / \mathrm{mol} \cdot \mathrm{K}$ and $T$ is the average absolute temperature of concrete $[\mathrm{K}] . E_{a}$ is often referred to as "apparent activation" energy as hydration is the combination of several physical and chemical phenomena overlapping in time, thus it does not show a constant value $[30,31]$. However, for defined ranges of hydration degrees (or strengths) it can be assessed as a single value. 
In this study the apparent activation energy was determined using the method of superposition which consists in adjusting the value of activation energy $E_{a}$, through Equations (7) and (8), in order to superpose the evolutions with time of the compressive strengths measured at $10{ }^{\circ} \mathrm{C}$ and $45^{\circ} \mathrm{C}$ with the reference evolution over an interval $\left[f_{c_{\min }} ; f_{c_{\max }}\right]$. The value of $E_{a}$ has been calculated by the method of least squares in order to provide the best overlap of the data sets corresponding to the three testing temperatures.

First the equivalent age of the three sets of specimens was assessed for the different temperatures of 10,20 , and $45{ }^{\circ} \mathrm{C}$. The specimens were stored at $20{ }^{\circ} \mathrm{C}$ during the first $24 \mathrm{~h}$ thus their common equivalent age at this time was 1 day. Then their equivalent ages were calculated using Equation (8). The values are given in Table 2. Their strengths at these equivalent ages were determined using Equation (6), with parameters $f_{c 28 \mathrm{~d}}=55.8$ and $s=0.21$, as equivalent age is the duration when concrete has to be stored at reference temperature (here $20^{\circ} \mathrm{C}$ ) to reach the same level of maturity. The value of $E_{a} \backslash R$ ratio was adjusted to minimize the differences between the measured strength and the calculated strength at each equivalent age. The values given in the Table 2 correspond to the best fit obtained with $E_{a} \backslash R=2936 \mathrm{~K}$. The highest strength value of $56.2 \mathrm{MPa}$ at $t_{e q}=60$ days was not taken into account as the method is not well suited for predicting the evolution of hydration and strength at long-term.

Table 2. Determination of apparent activation energy from compressive strengths.

\begin{tabular}{|c|c|c|c|c|}
\hline & Age & Equivalent Age & Strength $f_{c}^{T}$ & Strength $f_{c}^{T_{\text {ref }}}\left(t_{e q}(T)\right)$ \\
\hline Temperature & $t$ & $t_{e q}($ Equation (8)) & Compression Testing & Computation (Equation (6)) \\
\hline \multirow{4}{*}{$10^{\circ} \mathrm{C}$} & 1 & 1 & 19.7 & 22.3 \\
\hline & 2 & 1.7 & 25.6 & 29.1 \\
\hline & 7 & 5.21 & 37.4 & 42.1 \\
\hline & 28 & 19.95 & 53.2 & 53.6 \\
\hline \multirow{4}{*}{$20{ }^{\circ} \mathrm{C}$} & 1 & 1 & 19.7 & 22.3 \\
\hline & 2 & 2 & 31.1 & 31.1 \\
\hline & 7 & 7 & 42.8 & 45.1 \\
\hline & 28 & 28 & 55.8 & 55.8 \\
\hline \multirow{4}{*}{$45^{\circ} \mathrm{C}$} & 1 & 1 & 19.7 & 22.3 \\
\hline & 2 & 3.2 & 35.1 & 36.8 \\
\hline & 7 & 14.19 & 49.2 & 51.2 \\
\hline & 28 & 60.36 & 56.2 & 59.7 \\
\hline
\end{tabular}

\section{Results and Discussion}

\subsection{Assessment of the Operability of the Embedded Sensors}

The installation of a measuring system on a construction site in a moist environment is a challenging task. The risks of failure, particularly of the connectors, are significant. After the end of the construction stage, the number of defective sensors continues to increase. Failures are mainly caused by short circuits (leaking connections [26]). For the installation carried out on the on pile wharf, an assessment of the sensors in operation after one year is summarized in Table 3. 
Table 3. Sensors in operation.

\begin{tabular}{|c|c|c|}
\hline Type of Sensor & Number of Embedded Sensors & Number of Sensors in Operation \\
\hline FBG for strain measurement & 15 & 13 ( 2 broken wires) \\
\hline Resistivity & 4 & $\begin{array}{l}2 \text { with measurements fully available } \\
1 \text { with measurements partially available (water in connections) } \\
\qquad 1 \text { inoperable }\end{array}$ \\
\hline $\mathrm{RH} \%$ & 6 & 5 (broken wire) \\
\hline Pt 100 & 6 & 6 \\
\hline Reference electrode $\mathrm{Ag}+$ & 6 & 6 \\
\hline Thermocouples & 14 & 14 \\
\hline
\end{tabular}

In the resistivity sensor "partially operable" (RS 12), short-circuits occurred between some electrodes. These short-circuits are easy to identify since they give negative potentials or values close to zero. So they reduce the number of useable sequences of measurement and consequently induce an increase in the measurement error of the average resistivity.

The optical fiber partially operable is broken, so the light cannot be transmitted from one end to the other. This means that it cannot be interrogated using the amplified Brillouin scattering method which is the most sensitive Brillouin method. However, it can be probed by reflection from each end by Brillouin optical time domain reflectometry or optical Rayleigh back scattering.

In addition to the sensors broken during installation, the number of operational sensors will decrease over the years for many reasons. The life expectancy of the embeddable chloride depth electrode is certified 10 years as well as the reference electrode type Silver/Silver, Chloride/Potassium. Indeed, these sensors will stop working when all reagents are consumed.

For the other sensors, their life expectancy in a corrosive environment is not known. The estimation of the life expectancy of each sensor will be an important result of this project. However, the following events can be assumed:

- the filters of the humidity sensors may become clogged, preventing measurement (particularly due to the presence of chlorides);

- before this deadline, since these probes require recalibration every 2 years, the risk is that the sensor signal drifts slowly;

- Thermocouples type K and PT 100 probes are also susceptible to losing their calibration.

The drift of the measurements is a critical phenomenon since the measured temperature seems a priori correct. The drift of thermocouple measurements is, among other things, the result of the diffusion of atmospheric or metallic particles in thermocouple metals, most often caused by high temperatures. In the case of installation on a port wharf, the probable causes of measurement drift will be corrosion and strain-hardening of the sensors. These phenomena will also affect PT100 probes.

Finally, aberrant measures are likely to be recorded on all electrical sensors. Let us take the example of reference electrodes, These sensors should not normally be affected by external conditions since these sensors are designed to be embedded. However, the cable lengths are about $10 \mathrm{~m}$ and the measured potentials very low. Even with shielded cables, the low signal-to-noise ratio can have a negative influence on the measurement. Being able to identify sensors that provide incorrect information will be a major issue in this project for years to come.

Conversely, a long service life is expected for Bragg grating strain sensors. Since the information is coded in wavelength and not in amplitude, the signal should not drift. The measurement will therefore be usable as long as the optical fiber is mechanically protected by the stainless steel cladding. Thus, for future structural monitoring projects in harsh environments, it is expected that sensors based on fibre optic technologies will largely replace electrical sensors once the technology is available and affordable. This is, for example, what is emerging in the context of application to the monitoring of nuclear waste storage structures [32]. 


\subsection{Material Parameters}

The porosity of the concrete used on site was $12 \%$. This porosity value is relatively low [20] and in line with the class of concrete used during construction.

In this research, the interval $\left[f_{\mathcal{C}_{\min }} ; f_{\mathcal{C}_{\max }}\right]$ chosen to determine the activation energy, following Equation (8) was [29 MPa; $54 \mathrm{MPa}$ ]. Data are analyzed after the first $24 \mathrm{~h}$ during which all specimens are stored at the same temperature. Figures 10 and 11 show the time-temperature transformation based on the calculation of equivalent age. The bars on the graph correspond to the standard deviation of experimental measurements.

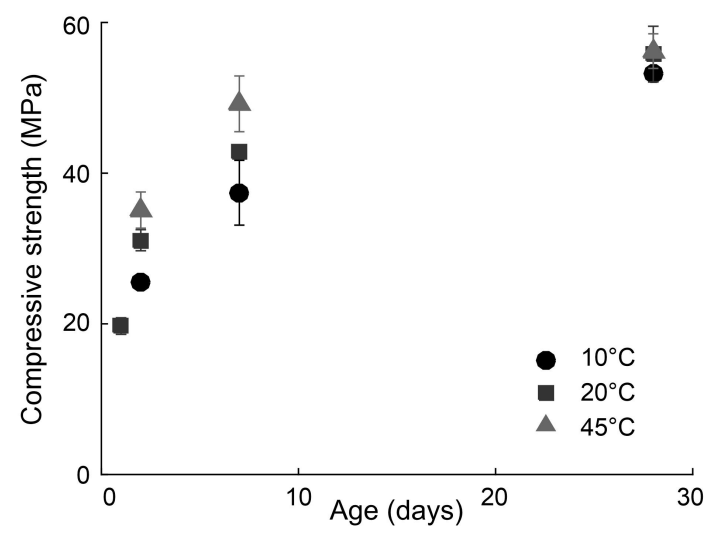

Figure 10. Influence of curing temperature on compressive strength.

The highest strength of 56.2 MPa resulted from 28 -day curing at $45^{\circ} \mathrm{C}$. The value of activation energy deduced from the superposition method was $24.4 \mathrm{~kJ} / \mathrm{mol}$. This value was used to assess equivalent time for the specimens cured at $10^{\circ} \mathrm{C}$ and $45^{\circ} \mathrm{C}$ on the graph of Figure 11.

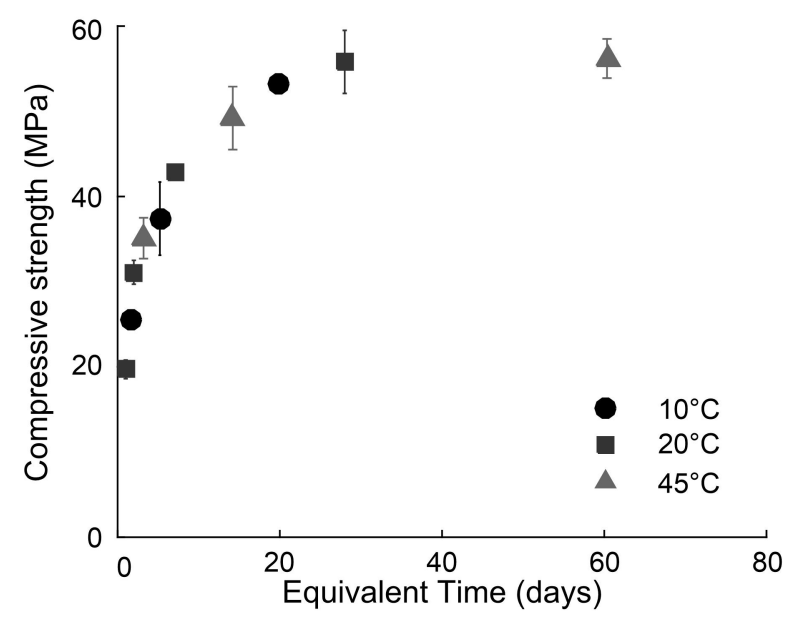

Figure 11. Compressive strength as a function of equivalent time.

Activation energy can be used to estimate concrete properties such as strength, modulus, and to a certain extent autogenous shrinkage $[33,34]$ at different locations of the studied beam from their temperature history measured in situ and the activation energy determined from compressive tests in laboratory (Figure 11). Yikici et al. [35] compared the strengths predicted by the maturity method and the actual strength measured on drilled cores. The predicted strength values were comparable to the core strength. Equations (6)-(8) have been used to assess the evolution of actual strength in the beam from the evolution of the actual temperature $T(t)$ (Figure 12). The Pt 14 sensor is located in the center on the beam, $\mathrm{Pt} 11$ at the bottom (Figure 7). The difference between both strength evolutions in 
relatively low, which is in line with relatively low temperature gradients measured across the section of the beam.

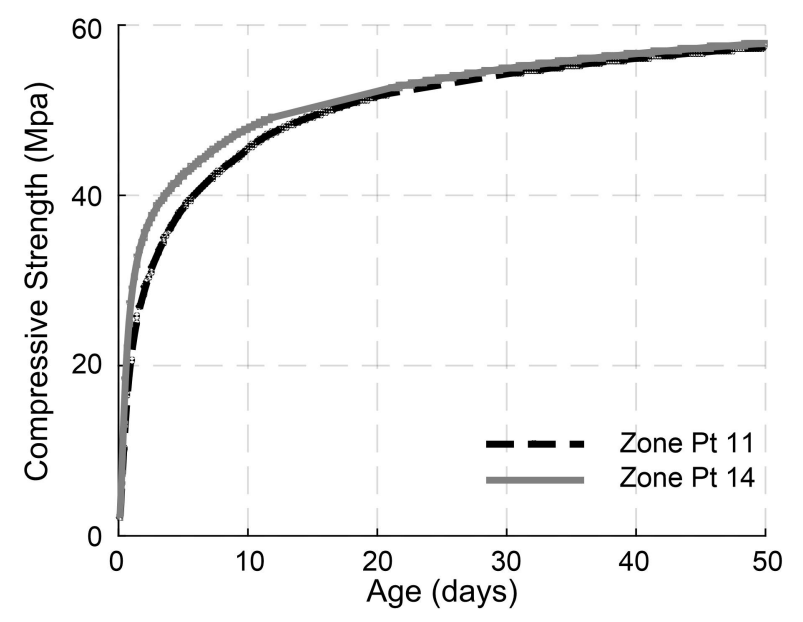

Figure 12. Modelling of concrete compressive strength evolution in beam 1 .

The evolution of Young's modulus provided by dynamic testing is plotted on the graph of Figure 13.

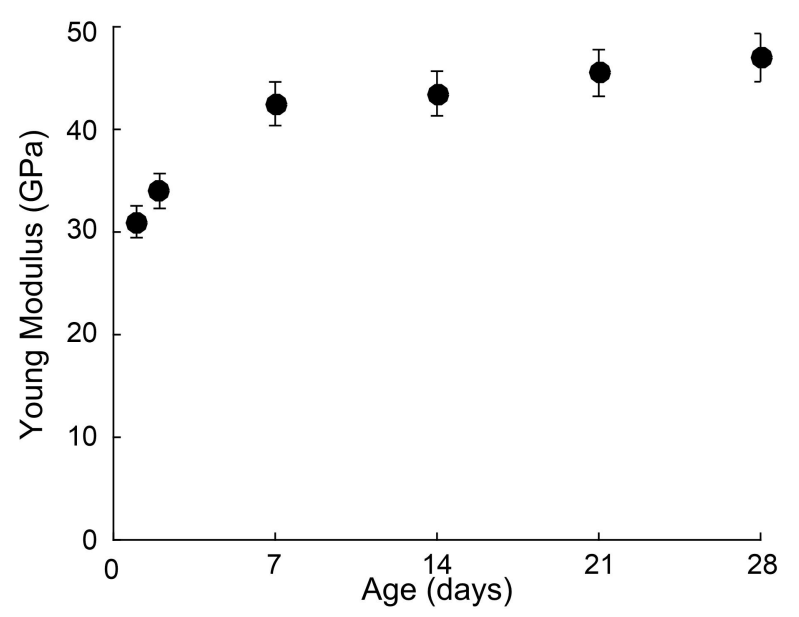

Figure 13. Elastic modulus of concrete specimens stored at $20^{\circ} \mathrm{C}$.

Although this method is known to overestimate this property [36], the experimental results show relatively high values of elastic modulus from the age of 1 day, which suggests that relatively low shrinkage or thermal strains are likely to generate significant stresses from early-age. Consequently, the shrinkage values and thermal gradients measured on site require a careful monitoring and analysis in relation to the mechanical resistances and the evolution of the modulus of elasticity.

\subsection{In Situ Measurement of Relative Humidity and Temperature}

The on-site temperature measurement is a mean for evaluating, the risks of cracking at early age, or the time required before removing the formwork [37,38]. In the present study, temperature measurement is an input for maturity method. Another use is the correction of the sensors' measurements, all sensitive to temperature (resistivity, strain and rebar potential). The different stages of the construction process are visible on the temperature evolution during the 60 first days (see Figure 14). The first peak on day 0 recorded by the two low level probes (Pt 11 and Pt 12) corresponds to the casting of the lower part of the beam. The second with a peak at $40^{\circ} \mathrm{C}$ for $\mathrm{Pt} 13$ (and $30^{\circ} \mathrm{C}$ for $\mathrm{Pt} 14$ ) corresponds to the casting of the central zone of the beam that affects also the 
temperature of the lower zone (peak at $20^{\circ} \mathrm{C}$ ). Finally, the peak at 16 days corresponds to the pouring of the slab.

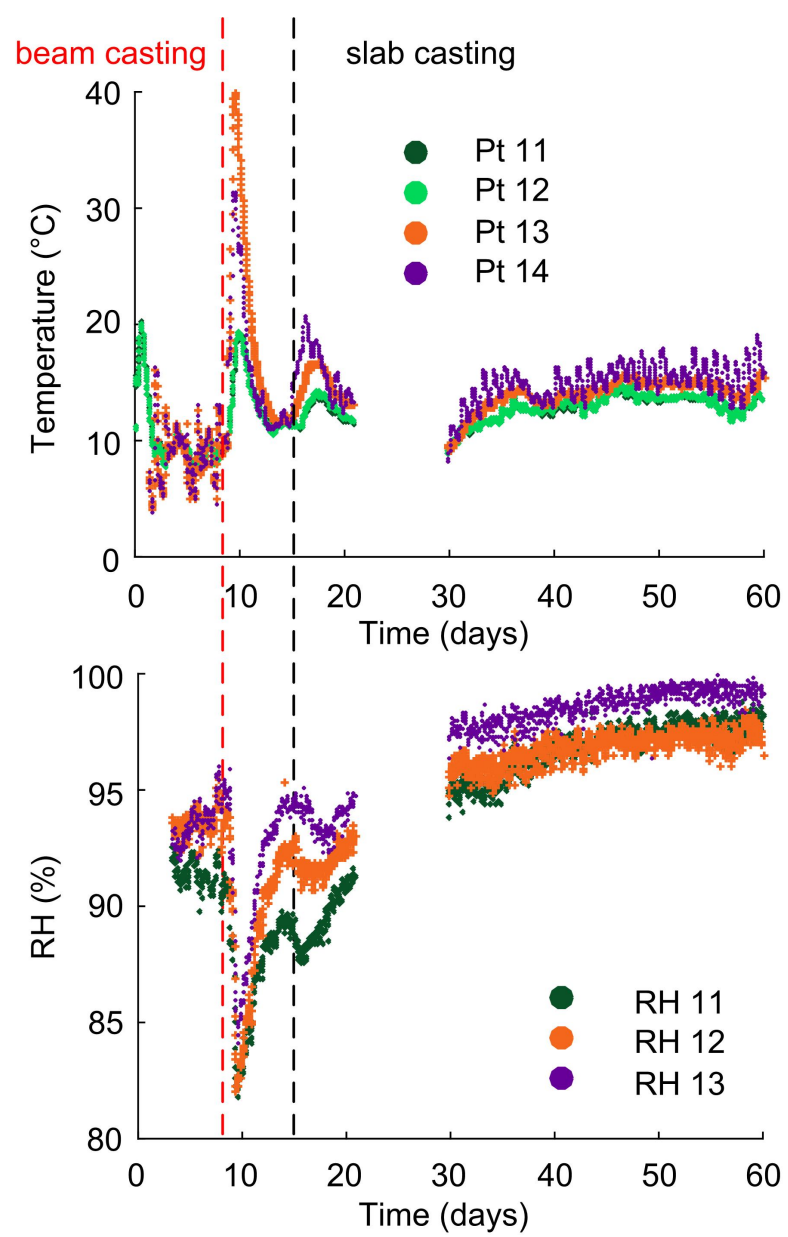

Figure 14. Temperature and relative humidity measured in beam 1 during the first two months.

Figure 15 gives the evolution of temperature during the first 12 months. Daily variations (day-night cycle) and seasonal variations are visible respectively on Figures 14 and 15. However, there is no visible variation linked to the tidal cycle.

High temperature at early age must be avoided as it is likely to trigger cracking during the first days and delayed ettringite formation at long term. Note that here the maximum temperature during beam casting did not exceed $40{ }^{\circ} \mathrm{C}$. Above $48{ }^{\circ} \mathrm{C}$, ettringite produced by the hydration of cement becomes less stable than monosulfate [39]. Thus the maximum temperature reached in the beam did not affect the stability of the main hydration products and delayed ettringite formation will be avoided [40]. Figure 14 also shows that the temperature gradients across the beam are acceptable in terms of thermal cracking mitigation. The maximum difference recorded between center and surface temperatures in beam 2 was approximately $10{ }^{\circ} \mathrm{C}$, which is low enough to prevent cracking due to internal restraint [41]. The measurements provided by the sensors thus confirm that the design of construction sequence was relevant to mitigate the pathologies due to temperature increase in concrete at early-age.

On the same beam, the relative humidity measured in concrete in the lower zone is shown in Figures 14 and 15. 

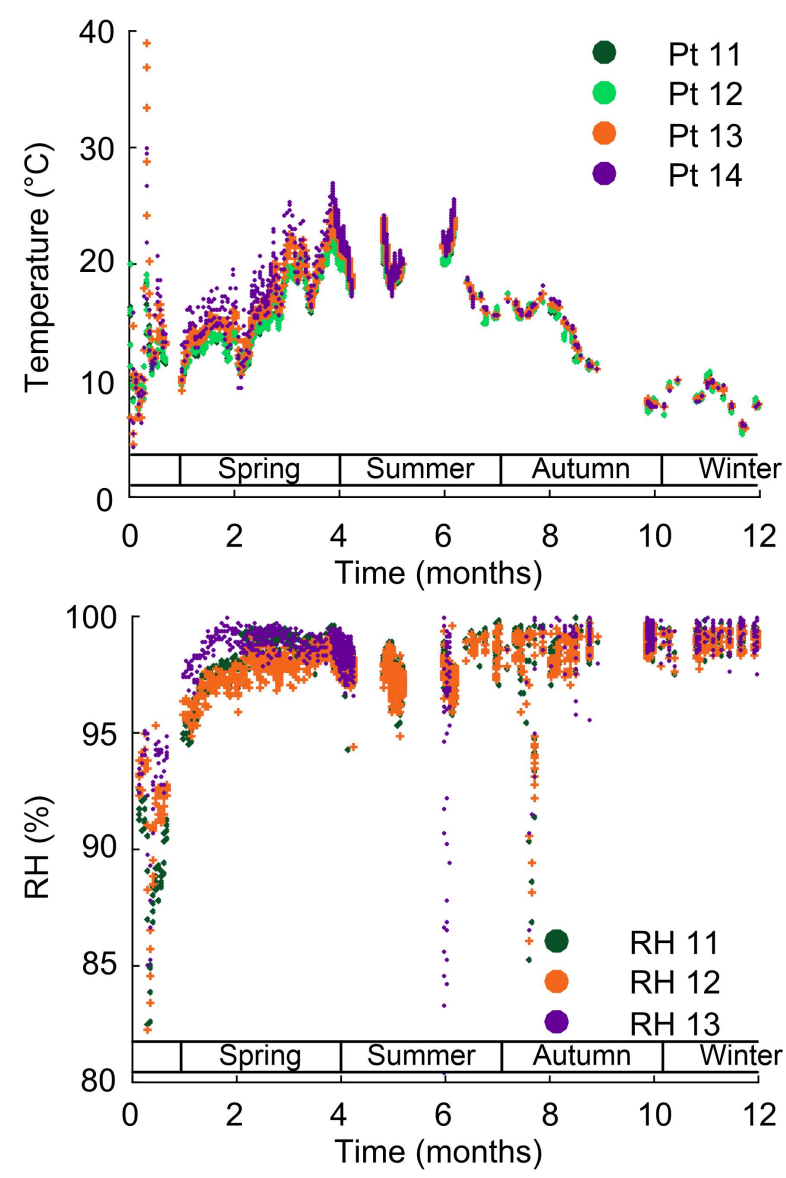

Figure 15. Temperature and relative humidity measured in beam 1 during the first year.

Recording began $48 \mathrm{~h}$ after the concrete was poured. The humidity probes are positioned at $80 \mathrm{~mm}$ depth in concrete openings recapped immediately after insertion of the sensors. It is therefore a measure of the relative humidity inside a cavity of a few cubic centimeters (about $10 \mathrm{~cm}^{3}$ ) in the concrete. This is not a direct measure of water content in the porosity. The relation between relative humidity measurement and concrete degree of saturation is not direct. It is given by the sorption-desorption isotherm of the studied concrete (see for example the studies of Harmathy [42] and Baroghel-Bouny [43]). Obtaining these curves requires maintaining samples in a controlled environment $\left(\mathrm{RH} \%\right.$ and $\left.\mathrm{T}^{\circ} \mathrm{C}\right)$ until the mass of the sample has stabilized and then gradually varying the relative humidity of the ambient air. These measurements are not complex but require several months or years of testing since the time required to stabilize the samples is very long. This time is comparable to hydration characteristic times thus both phenomena are coupled during the period of interest, and it is not possible to deduce the water saturation from relative humidity measurements since casting time. The desorption curves published in the literature show that a concrete subjected over the long term to a very humid environment (between 98 and $100 \%$ of relative humidity) is nearly saturated with water. Several factors actually influence concrete saturation, mainly hydration and external drying. Given the water-to-cement ratio of 0.45 (see Table 1), free water remains in the pores even after the complete hydration of cement [44]. The decrease of relative humidity in concrete due to external drying is a relatively slow phenomenon, even at a depth of a few centimeters (see [45]). Given the stability of RH over the long term (here between $98 \%$ and $100 \%$ of relative humidity), the $\mathrm{RH}$ of studied beam was still not affected by external drying. The underside elements of the structure are regularly submerged or splashed during tidal cycles. A decrease of this relative humidity to 95\% after four months corresponds to the summer season with higher temperature in concrete (see Figure 15 where the 5th month corresponds to the beginning of July 2017).The relatively humidity in 
concrete bean was actually affected by the diffusion of heat as the decreases of relative humidity can be correlated with temperature peaks (see Figure 15). Although it is not possible to state that concrete is saturated with water only on the basis of a relative humidity measurement in a cavity, the water saturation of concrete is likely very high. This assumption is consistent with the low resistivity values measured on site (see Section 4.5) while the porosity is low (see Section 4.2). The underside elements of the structure are regularly submerged or splashed during tidal cycles which probably explains this relative humidity value in the concrete.

Alternative measurement methods could also have been used to directly measure the degree of saturation of a given concrete after a calibration operation. Resistivity measurement is an example, but capacitance probes or Time-Domain Reflectometer (TDR) probes can also be mentioned. The disadvantage of these technologies lies in their sensitivity to pollutants, particularly chlorides.

\subsection{Strain Measurement}

The shrinkage measurements (considering correction of thermal effect and equivalent age of concrete) are presented for the sensors located on the middle level in Figure 16. The extensometer supporting frame 1 is near the bank and the 5 on the river side (see Figure 6). For the sensors of the high and low level, plots are given in the appendix because the trends are similar. The mean value of each level is also considered for analysis. Similar shrinkage rates can be observed for the different levels (low, middle and top in Appendix B). The shrinkage was systematically higher in the center of the beam, in the most massive part. In absence of service loading, the strain measurement could appear of fair interest. Nevertheless, after taking into account the temperature evolution and once associated with the results of the material tests (modulus, strength, shrinkage in laboratory), shrinkage is one of the main parameters affecting the risk of cracking at early age.

The strain of concrete at $20^{\circ} \mathrm{C}$ in laboratory conditions has been plotted on Figures 16 and 17 as a function of equivalent time. This takes into account the influence of measured temperature on equivalent age of concrete (see Section 3). Equivalent age is often used to predict the actual strength of concrete knowing its temperature history. This concept has been used for the prediction of autogenous shrinkage [33]. These authors actually showed that maturity can be used for isothermal and realistic conditions as long as temperatures remain between 10 and $40{ }^{\circ} \mathrm{C}$. The conditions of the evolution of the structural concrete can be considered as similar to autogenous shrinkage as the relative humidity in concrete remained close to $100 \%$ (see Figures 14 and 15). This allowed the hydration of cement to continue, thus the observed shrinkage was mainly due to self-desiccation. The strains obtained from laboratory experiments are of the same order of magnitude as the values determined from the measurements on the construction site. The $100 \mu \mathrm{m} / \mathrm{m}$ is the typical magnitude of the autogenous shrinkage of normal strength concrete.

In both series of results (laboratory and in situ) a slight swelling can be observed during the first 20 days. This cannot be attributed to thermal dilation as the peak due to hydration appeared much earlier. Casting took place at 8.9 days and the temperature actually came back to casting temperature at 11.7 days (Figure 14). This swelling has already been observed in previous experimental studies [46]. This could be due to the rapid growth of hydration products at early age and the resulting crystallization pressure exerted on the pore walls [47-49]. 


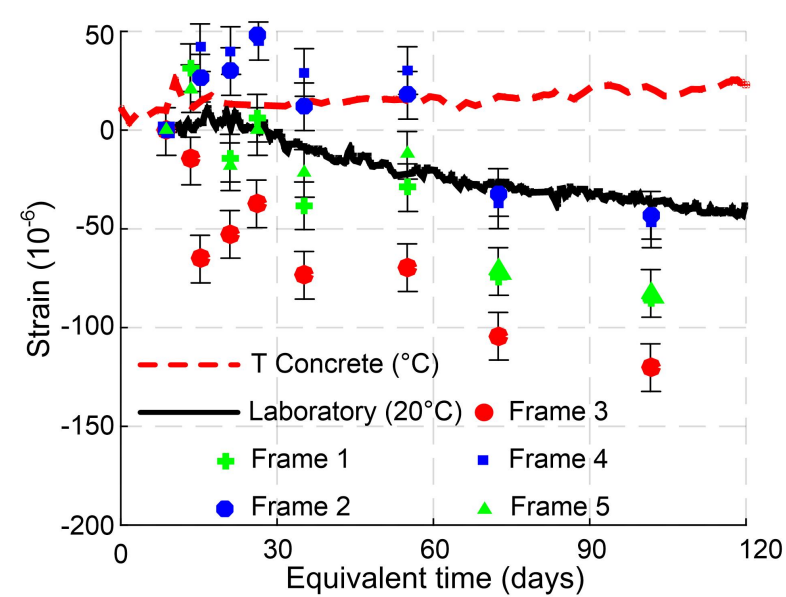

Figure 16. Temperature (dashed red line), strain of structural concrete in the middle level of frames 1 to 5 and strain at the material scale from laboratory measurement (solid black line).

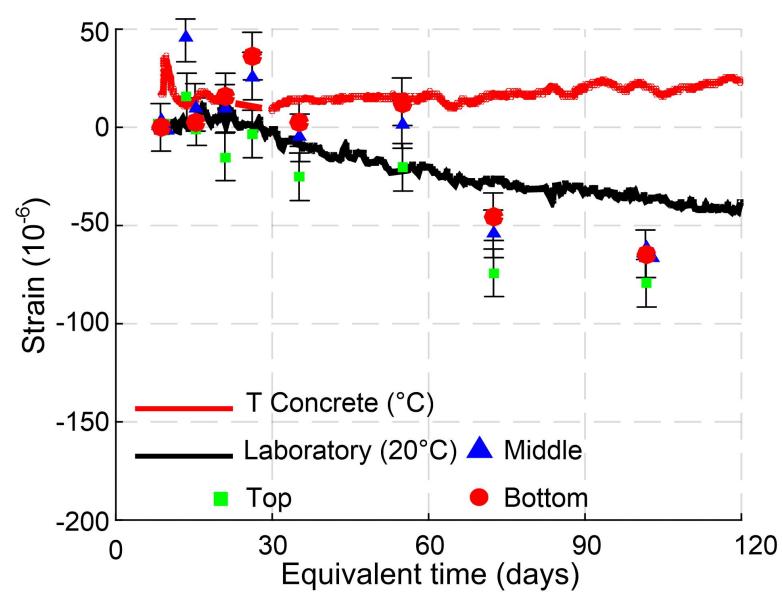

Figure 17. Temperature (solid red line), average strain over four frames of structural concrete at the top, middle and bottom levels of the frames (average value of extensometers located on the supporting frames 1, 2, 4 and 5) and strain at the material scale from laboratory measurement (solid black line).

Sensors located on the supporting frame 3 showed higher deformation. They were located in the center of the beam, where the temperature increase was the highest according to the measurement performed in beam 2 (see Figure 18). Peak temperature was $34{ }^{\circ} \mathrm{C}$ in the center and between 27 and $31{ }^{\circ} \mathrm{C}$ in the four other locations (see Figures 7 and 18). The temperature decrease causes the buildup of tensile stresses due to the increase of elastic modulus during concrete hardening, and the restraint is higher in the central part, thus higher tensile stress could be expected at frame 3 . The higher values given by sensors located on the supporting frame 3 would result not only from material parameter related to autogenous shrinkage, but also from structural effect. The evolutions of the deformations at the four other locations were averaged and plotted on the same graph on Figure 17 as the deformation measured in the laboratory. Both series of data are globally in good agreement. Lower values are given by the sensors located at the top of the beam. This can be interpreted by the effect of compressive load induced by bending. 


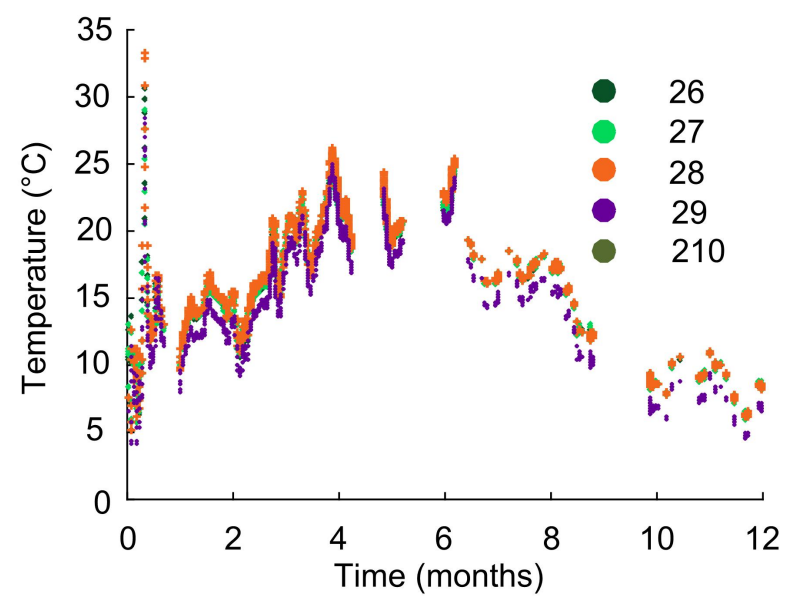

Figure 18. Measurement of the temperature using thermocouples sensors in beam 2 at the locations of the strain sensors on beam 1 .

\subsection{Measurement of Apparent Resistivity}

Resistivity measurement is plotted on a map of apparent resistivity of the area between the sensor and the surface exposed to the sea. Figure 19 presents an example of resistivity map measured in beam 1 with sensor RS21, respectively 148 and 300 days after casting in concrete. In this case, the resistivity is about $51 \Omega \cdot \mathrm{m}$ with erratic variations between $47 \Omega \cdot \mathrm{m}$ and $55 \Omega \cdot \mathrm{m}$ for the measurement performed the day 148 while it is about $62 \Omega \cdot \mathrm{m}$ with erratic variations between $57 \Omega \cdot \mathrm{m}$ and $66 \Omega \cdot \mathrm{m}$ for the measurement performed the day 300 .

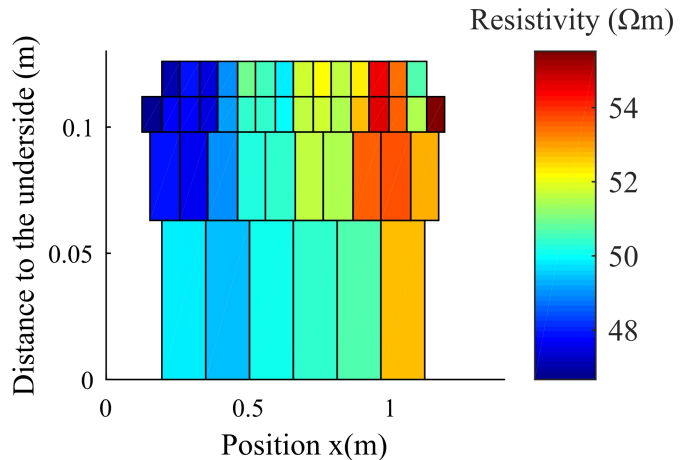

a) Map: RS21 day 148

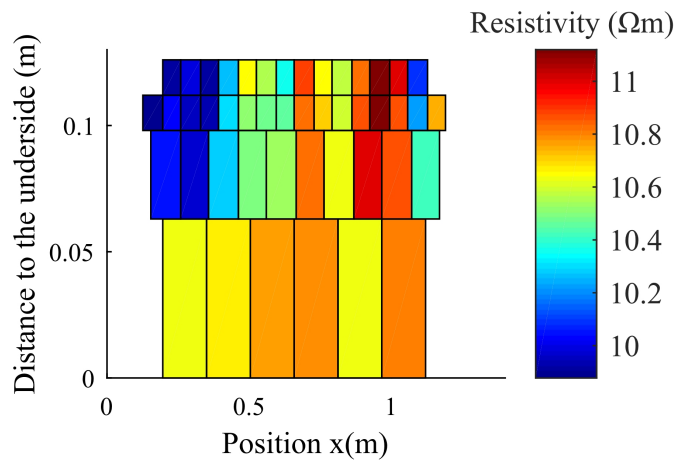

c) Difference between days 148 and 300

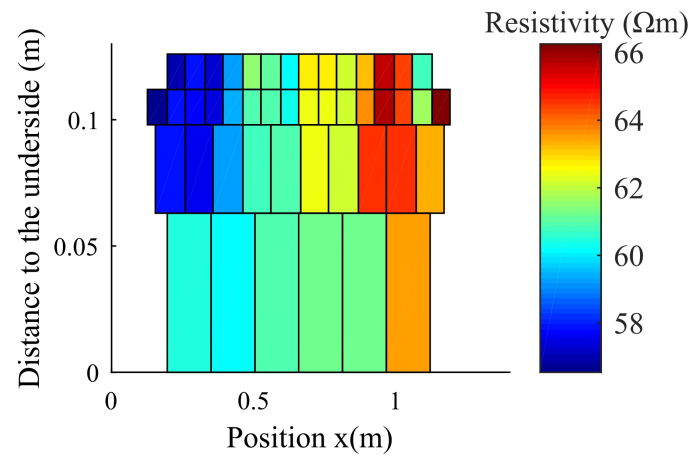

b) Map: RS21 day 300

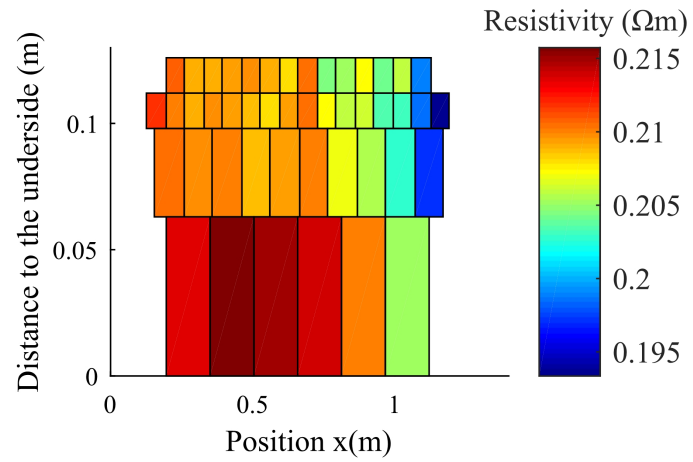

d) Relative difference between days 148 and 300

Figure 19. Example of pseudo section of apparent resistivity measured in beam 1 by sensor RS 11, 148 days after casting in concrete (a), 300 days after casting in concrete (b), (c) is the difference between the two pseudo sections of resistivity and (d) the relative difference. 
The apparent resistivity depends mainly on the temperature, the porosity of the medium, the electrolyte content of the pores and the conductivity of the porous solution. By comparing the pseudo section measured at different dates, it will be possible to detect a drop in resistivity and the probable progress of a chloride front. An example is provided in Figure 19b,c. In this case the difference is positive, which indicates a possible drying of the concrete.

At early age, the chloride ions have not significantly penetrated inside the beams. The spatial dispersion of measured resistivity is mainly due to the heterogeneity of the material. It is then reasonable within the framework of process monitoring to use the mean value of the measured resistivity as the value of the global resistivity of concrete. An example is given for beam 1 in Figure 20. Each point on this curve represents the average of the 129 resistivity values measured on site at a given equivalent age (according to the computation explained in Section 3.3). The error bar represents the standard deviation of the resistivity values around the average value of the measurements taken for a sensor. Finally, since the resistivity changes with temperature, a thermal correction was performed to compute the resistivity for the reference temperature $T_{\text {ref }}=20^{\circ} \mathrm{C}$ according to Equation (9):

$$
\rho_{T_{\text {mes }}}=\frac{\rho_{T_{\text {ref }}}}{1+\alpha\left(T_{\text {mes }}-T_{\text {ref }}\right)}
$$

where $\rho_{T_{\text {mes }}}$ is the apparent resistivity measured at the temperature $T_{\text {mes }}$ and $\rho_{T_{\text {ref }}}$ is the apparent resistivity computed for the temperature $T_{\text {ref. }}$. Finally, $\alpha$ is the correction factor of temperature effects. In this study, the coefficient is set at $0.022{ }^{\circ} \mathrm{C}^{-1}$ as proposed by Whittington and al. [50].

The corrected curves are shown on Figure 20a. With the thermal correction, the curves are similar to those describe by the authors who performed resistivity measurement in laboratory on concrete specimens, most often with Wenner portable devices [13,51]. This result validates the relevance of the measurements obtained with the in-situ device. Moreover, the two sensors embedded in beam 2 (RS21 and RS 22) about $20 \mathrm{~cm}$ apart and $1 \mathrm{~m}$ long each, give almost the same average values.

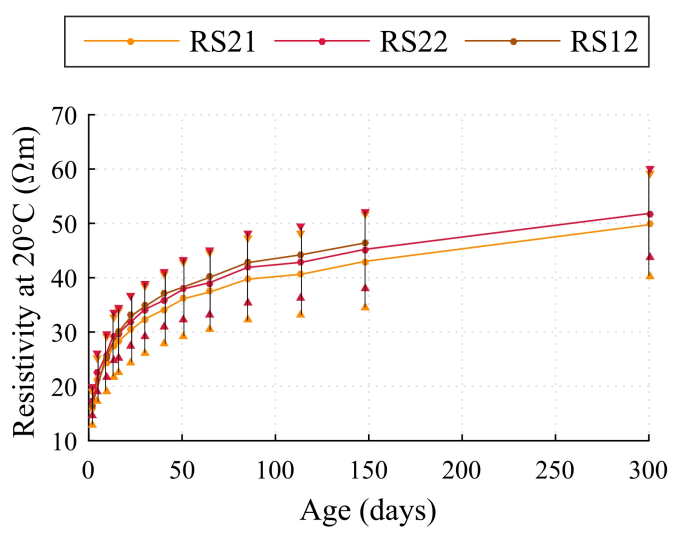

a) Apparent resistivity

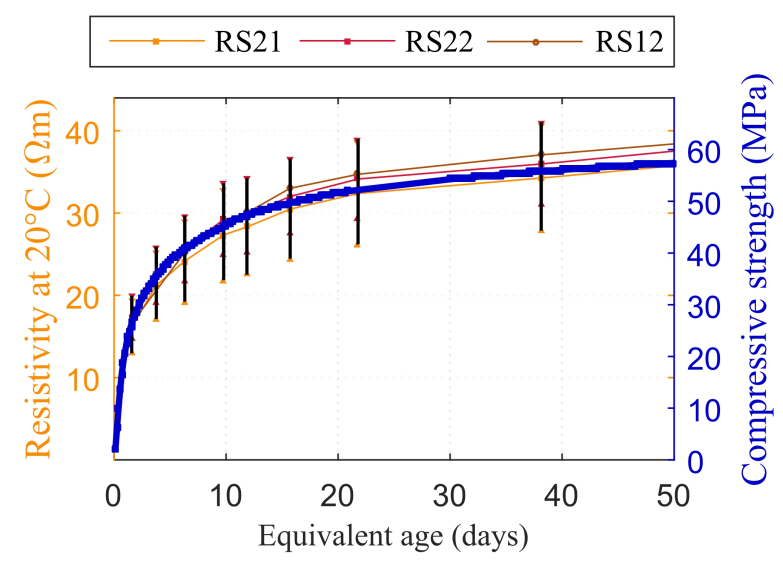

b) Apparent resistivity and compressive strength

Figure 20. Evolution of the average value of the apparent resistivity computed at $20{ }^{\circ} \mathrm{C}$ vs concrete age [0-300 days] (a) and apparent resistivity computed at $20^{\circ} \mathrm{C}$ vs. concrete equivalent age [0-50 days] (b) plotted with evolution of compressive strength. Error bars represent the standard deviation $\sigma$ of measurements.

As shown in Figure 20b, there is a strong correlation between resistivity and compressive strength evolution. The resistivity of concrete is dependent on many factors, e.g., pore structure, pore solution, temperature and degree of water saturation. However, for a given concrete (same chemical composition), whose degree of saturation remains stable and after a correction of thermal variation on measurement, the only variable parameter is the porosity. In this study, it is reasonable to consider that the degree of hydration changes only slightly (see the value of $\mathrm{RH} \%$ always close to 
$100 \%$ during the first year of structure service life). Additionally, it is recognized that the strength of concrete is mainly determined by porosity instead of other factors, e.g., pore solution and water saturation. Since the porosity evolves with hydration [52] thus modifying the resistivity value and the mechanical strength of concrete, the resistivity measurement can be considered as an indicator of material hardening. As a consequence, when the resistivity has reached its asymptotical value, it is probable that compressive strength is close to the maximum.

\subsection{Discussion}

The analysis of the experimental data collected in-situ shows that to obtain exploitable information, it is necessary to analyze simultaneously measurements from the various sensors and the results of tests carried out in laboratory. With the exception of temperature, data from a single type of sensor does not contain information that can be used directly to monitor manufacturing processes. An example is the strain measurement. Combined with temperature measurement and material testing, it can detect areas where concrete shrinkage is likely to cause cracking of the structure at early age. It is easier to estimate the behavior of concrete from laboratory tests. The humidity measurements performed in situ confirmed that the shrinkage was mainly due to self-desiccation, thus laboratory tests in sealed conditions can be considered as relevant.

In addition to the simultaneous analysis of several measurands, it is important to underline the importance of the measurement history. The knowledge of the initial state of each sensor is particularly crucial. This is the case, for example, for calculating concrete shrinkage based on strain measurements. More generally, a continuous or regular measurement is easier to interpret than a punctual measurement giving the state of the structure. Whenever possible, it is therefore interesting to start data acquisition before concreting.

The resistivity measurement provides another illustration. In the scientific literature, a threshold value is retained as an indicator of corrosion. The probability of initiation of corrosion is considered high for a value of $100 \Omega \cdot \mathrm{m}$ [13]. Within the wharf, on a new structure, and after several months of measurement, the resistivity is still below this threshold. As explained above, since concrete is not very porous, only a high water content of the material, due to the location of the structure (on the riverside) can explain this measure. To have relevant information on the evolution of the corrosion risk and to be able to detect the progress of the chloride front in a particular area, the exploitable information is the relative difference between two states [53], i.e., between two pseudo sections of apparent resistivity as presented in Figure 19. If this relative difference shows a decreasing resistivity value near the surface of the structure, one possible conclusion is that it is caused by the penetration of a chloride front. A strong correlation was observed between increased resistivity and mechanical strength. The study of Liu et al. [54] actually showed that after the de-percolation time of capillary pores, the resistivity of cement paste is controlled by the volume fraction of $\mathrm{C}-\mathrm{S}-\mathrm{H}$, which is also the main hydration product for its contribution to mechanical strength. The measurement of resistivity could thus be used as part of material monitoring. The "ageing factor" which characterizes the time evolution of the chloride diffusion coefficient can be determined from resistivity measurements on saturated specimens [55]. The resistivity has the advantage of being a non-destructive technique, thus it allows a continuous monitoring in the same specimen (laboratory) or at the same location of a structure (in-situ). The maturity analysis and the determination of activation energy allow correlating the evolutions of strength, resistivity, and temperature.

\section{Conclusions}

This article relates the instrumentation with a multi-sensor measurement chain of a wharf in Saint-Nazaire, France. The structural health monitoring system had two objectives: on the one hand, to contribute to feedback on the operation of the structure in service and, on the other hand, to extend the service life of the structure. The monitoring started in the instrumented area at the same time as the in-situ concreting. The analysis of the parameters assumed as the most sensitive to concrete 
changes, and therefore to the manufacturing process, was thus carried out during the first months of the structure's life thanks to the following sensors:

- $\quad$ Fiber optic extensometers (FBG);

- Pt 100 and thermocouple sensors;

- Relative humidity sensors;

- Embedded resistivity sensors.

These measurements were supplemented by material testing in the laboratory: 40 samples were casted with the concrete used in the instrumented beams and submitted to several mechanical tests. The porosity of the concrete was found equal to $12 \%$. Porosity value can be considered as low [20]. This is consistent with the use of high performance concrete with high mechanical strength. The compressive strength was close to $60 \mathrm{MPa}$ after 50 days. The Young modulus was greater than $30 \mathrm{GPa}$ after 1 day and tended to $45 \mathrm{GPa}$. Significant stress is likely to appear at early age due to this high value of Young Modulus, which reinforces the need for a careful monitoring. The construction sequence allowed mitigating the early-age thermal cracking as the maximum temperature and the thermal gradient did not exceed $40{ }^{\circ} \mathrm{C}$ and $30^{\circ} \mathrm{C}$ respectively.

After one year, 53 sensors over 59 are fully operable, and 2 are partially operable. This has made possible a thorough characterization of the evolution of the structure at early stage. First of all, the relative humidity measured in small cavities inside the beams remains close to $98 \%$ during the first year. This slow evolution is probably due to the fact that the lower part of the wharf is cyclically submerged. The temperature exhibits a peak at $40{ }^{\circ} \mathrm{C}$ in the center of the beams during casting. Then it is mainly driven by external temperature. Seasonal and daily variations were observed but no variation linked to the tidal cycle. The in-situ strain measurements show that the extremities of the beam experience a strain similar to the autogenous strain measured in laboratory, whereas the central part is twice strained. This higher value of strain is influenced by structural effects. At early age, the resistivity of the concrete is almost uniform and remains lower than $70 \Omega \cdot \mathrm{m}$. This value is smaller than the value of $100 \Omega \cdot \mathrm{m}$ classically considered as the threshold value corresponding to high probability of corrosion. Beyond this risk of corrosion due to the environment of the structure, it will be interesting to compare the resistivity values at different times, to be able to identify a possible modification of the corrosion risk. Calculating the difference between two pseudo-sections of apparent resistivity will thus be a method allowing the detection of an area potentially contaminated with chloride ions. Moreover, it has been shown that the resistivity is a good indicator of material hardening. It indicates especially when the concrete has reached its long-term compressive strength.

The simultaneous start of the acquisition with concreting made it possible to validate the operation of the measuring chain and the identification of the malfunction of failed sensors. Analysis of the data at early age showed that strain and resistivity measurements could be directly exploited within the framework of manufacturing process monitoring. However, to be fully exploitable, these measurements must be combined with temperature measurements. Moisture measurements and material testing also provide interesting additional information for analysis. Strength measurements combined with the determination of activation energy, and the monitoring of resistivity, allow comparing the development of mechanical and physical concrete properties and showing their correlation.

The study presented here proposes a multi-physical database of in situ measurements or material tests collected during one year. The initial state of the structure and the material are therefore perfectly known. The data presented in this paper is meant to be updated within five to 10 years once the degradation process has begun. The knowledge of the complete measurement history will promote a better understanding of degradation processes. Additionally, the study shows that a measurement chain dedicated to the SHM could be a tool for validating the quality of the construction of a reinforced concrete structure. The measuring chain allows early detection of structural disorders, thus limiting the risk of costly repairs during the operating phase. 
The data collected on site and the results of the material tests presented in this article are available in the Supplementary Materials. The aim is to propose a database to analyze the link between measurements carried out in laboratory with data recorded during the construction of a real structure.

Supplementary Materials: The Supplementary Materials are available online at http:/ /www.mdpi.com/20771312/7/4/84/s1.

Author Contributions: Conceptualization, Y.L. and E.R.; Funding acquisition, F.S.; Investigation, M.C., V.G., R.G., Y.L., D.L., C.L., J.P. and E.R.; Project administration, Y.L. and F.S.; Writing—original draft; V.G., Y.L., D.L., J.P. and E.R.; Writing - review \& editing M.C., C.L. and F.S.

Funding: This research was funded by the Pays de la Loire region within the framework of IMARECO2 project.

Acknowledgments: The authors would like to thank all the partners of iMAREO2 project: Keops Automation (D. Follut, D. Olivier), Université de Nantes (M. Roche), Nantes-Saint Nazaire Port (P. Lijour, M. Labegorre) and Bouygues Construction (the teams of Bouygues Travaux Publics Régions France). Special thanks are due to Thierry Giorgetti (acquisition unit installation) and Antoine Bassil (sensors installation) for their help in this project. The authors would like to thank the Pays de la Loire region for its financial support.

Conflicts of Interest: The authors declare no conflict of interest.

\section{Appendix A. Sensor References}

Table A1. Sensor used on site: references and suppliers.

\begin{tabular}{ccc}
\hline Sensor & Reference & Supplier \\
\hline Embedded Strain Sensor & FS62 length 100 mm & HBM \\
Optical cable & BRUsens DSS 2.8 mm V1 non-metallic & SOLIFOS \\
$\mathrm{T}{ }^{\circ}$ C and RH $\%$ probes & HC2-C05 & ROTRONIC \\
PT 100 probe & ROF-PT100 Class A & ROTRONIC \\
Embeddable chloride depth electrode & CP80 & C-Probe Systems \\
Reference electrode & CP10P Electrode & C-Probe Systems \\
Silver/Silver Chloride/Potassium & & \\
\hline
\end{tabular}

\section{Appendix B. Strain Measurement in Laboratory and Beam 1}

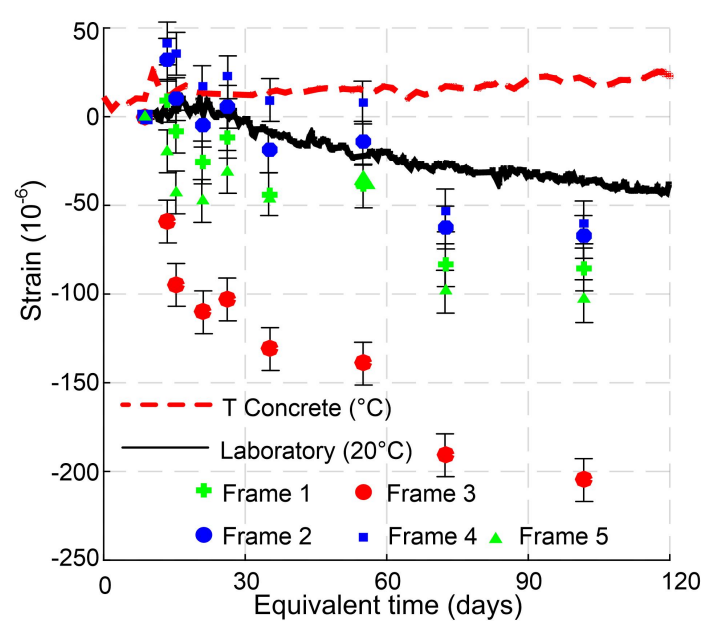

(a)

Figure A1. Cont. 


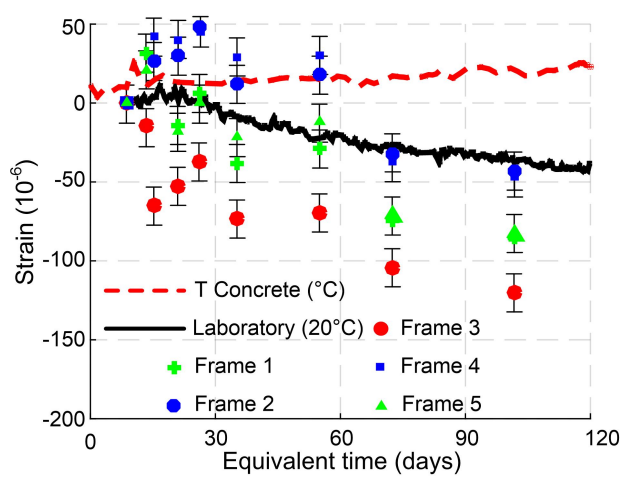

(b)

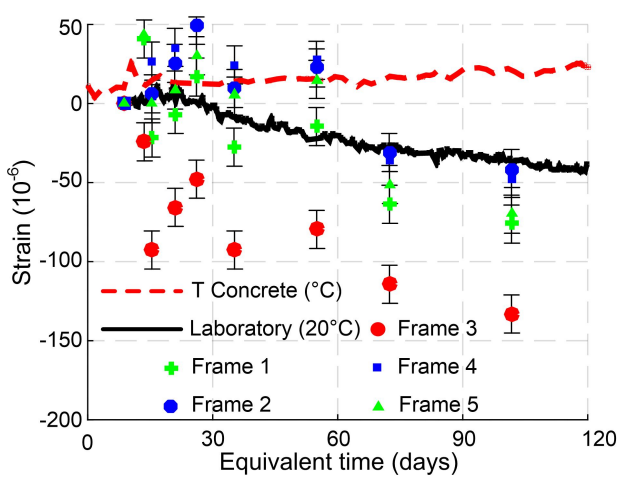

(c)

Figure A1. Strain measurement in concrete during concrete shrinkage for the whole sensors. (a) Strain measurement in the top level; (b) Strain measurement in the middle level; (c) Strain measurement in the bottom level.

\section{References}

1. Boero, J.; Schoefs, F.; Capra, B.; Rouxel, N. Technical management of French harbour structures-Part 1: Description of built assets. Rev. Paralia 2009, 2, 6.1-6.11. [CrossRef]

2. Boero, J.; Schoefs, F.; Capra, B.; Rouxel, N. Technical management of French harbour structures—Part 2: Current practices, needs-Experience feedback of owners. Rev. Paralia 2009, 2, 7.1-7.12. [CrossRef]

3. von Wolffersdorff, P. Workshop Sheet Pile Test Karlsruhe; Technical Report; Delft University: Delft, The Netherlands, 1994.

4. Finno, R.J.; Roboski, J.F. Three-Dimensional Responses of a Tied-Back Excavation through Clay. J. Geotech. Geoenviron. Eng. 2005, 131, 273-282. [CrossRef]

5. Del Grosso, A.; Lanata, F.; Brunetti, G.; Pieracci, A. Structural health monitoring of harbour piers. In Proceedings of the 3rd International Conference on Structural Health Monitoring of Intelligent Infrastructure, Vancouver, BC, Canada, 13-16 November 2007.

6. Catbas, F.N.; Aktan, A.E. Condition and Damage Assessment: Issues and Some Promising Indices. J. Struct. Eng. 2002, 128, 1026-1036.:8(1026). [CrossRef]

7. Delattre, L.; Duca, V.; Sherrer, P.; Rivière, P. Anchoring efforts on Osaka quay wall at LeHavre harbour. In Geotechnical Engineering for Transportation Infrastructure; Balkema, A.A., Ed.; A.A. Balkema: Rotterdam, The Netherlands, 1999; Volume 2, pp. 713-718.

8. Schoefs, F.; Yáñez-Godoy, H.; Lanata, F. Polynomial Chaos Representation for Identification of Mechanical Characteristics of Instrumented Structures. Comput. Aided Civ. Infrastruct. Eng. 2011, 26, 173-189. [CrossRef]

9. Schoefs, F.; Le, K.T.; Lanata, F. Surface response meta-models for the assessment of embankment frictional angle stochastic properties from monitoring data: An application to harbour structures. Comput. Geotech. 2013, 53, 122-132. [CrossRef] 
10. Liu, H.B.; Zhang, Q.; Zhang, B.H. Structural health monitoring of a newly built high-piled wharf in a harbor with fiber Bragg grating sensor technology: Design and deployment. Smart Struct. Syst. 2017, 20, 163-173. [CrossRef]

11. Lecieux, Y.; Schoefs, F.; Bonnet, S.; Lecieux, T.; Lopes, S.P. Quantification and uncertainty analysis of a structural monitoring device: Detection of chloride in concrete using DC electrical resistivity measurement. Nondestruct. Test. Eval. 2015, 30, 216-232. [CrossRef]

12. Saleem, M.; Shameem, M.; Hussain, S.E.; Maslehuddin, M. Effect of moisture, chloride and sulphate contamination on the electrical resistivity of Portland cement concrete. Construct. Build. Mater. 1996, 10, 209-214. [CrossRef]

13. Polder, R.B. Test methods for on site measurement of resistivity of concrete-A RILEM TC-154 technical recommendation. Construct. Build. Mater. 2001, 15, 125-131. [CrossRef]

14. Morris, W.; Vico, A.; Vazquez, M.; de Sanchez, S.R. Corrosion of reinforcing steel evaluated by means of concrete resistivity measurements. Corros. Sci. 2002, 44, 81-99. [CrossRef]

15. Andrade, C.; d'Andrea, R.; Rebolledo, N. Chloride ion penetration in concrete: The reaction factor in the electrical resistivity model. Cem. Concr. Compos. 2014, 47, 41-46. [CrossRef]

16. AFNOR. Mesure de l'Humidité de l'air-NF X15-119; Technical Report NF X15-119; AFNOR: Paris, France, 1999.

17. du Plooy, R.; Palma Lopes, S.; Villain, G.; Dérobert, X. Development of a multi-ring resistivity cell and multi-electrode resistivity probe for investigation of cover concrete condition. NDT E Int. 2013, 54, 27-36. [CrossRef]

18. LCPC. Guide Technique La Résistance du Béton Dans L'ouvrage; Technical Report; LCPC: Chapel Hill, NC, USA, 2003.

19. Samouh, H.; Rozière, E.; Wisniewski, V.; Loukili, A. Consequences of longer sealed curing on drying shrinkage, cracking and carbonation of concrete. Cem. Concr. Res. 2017, 95, 117-131. [CrossRef]

20. Baroghel-Bouny, V. Conception Des Bétons Pour une Durée de Vie Donnée des Ouvrages; Technical Report; Association Française de Génie Civil: Paris, France, 2004.

21. AFNOR. Essai Pour Béton Durci-Essai de Porosité et de Masse Volumique-NF P18-459; Technical Report NF P18-459; AFNOR: Paris, France, 2010.

22. Spinner, S.; Tefft, W. A Method for Determining Mechanical Resonance Frequencies and for Calculating Elastic Moduli from These Frequencies; ASTM: West Conshohocken, PA, USA, 1961; pp. 1221-1238.

23. Jensen, O.M.; Hansen, P.F. Autogenous deformation and RH-change in perspective. Cem. Concr. Res. 2001, 31, 1859-1865. [CrossRef]

24. Ferdinand, P. Capteurs à Fibres Optiques à Réseaux de Bragg. Available online: https: / / www.techniques-ingenieur.fr/base-documentaire/mesures-analyses-th1/cnd-methodes-surfaciques42586210/ capteurs-a-fibres-optiques-a-reseaux-de-bragg-r6735/ (accessed on 27 March 2019).

25. Wenner, F. A method of measuring earth resistivity. J. Wash. Acad. Sci.1915, 5, 561-563. [CrossRef]

26. Barker, R. Depth of investigation of collinear symmetrical four-electrode arrays. Geophysics 1989, 54, 1031-1037. [CrossRef]

27. Lanata, F.; Schoefs, F. Multi-algorithm approach for identification of structural behavior of complex structures under cyclic environmental loading. Struct. Health Monit. 2011. [CrossRef]

28. CEN. NF EN 1992-1-2-Octobre 2005-Calcul des Structures en béton-Partie 1-1: Règles Générales et Règles Pour les Batiments; Technical Report; CEN: Bruxelles, Belgium, 2005. (In French)

29. Freiesleben Hansen, P.; Pedersen, E. Maleinstrument til Kontrol af betons haerdning. Nordisk Betong 1977, 1, 21-25.

30. D'Aloia, L.; Chanvillard, G. Determining the "apparent" activation energy of concrete: Ea-Numerical simulations of the heat of hydration of cement. Cem. Concr. Res. 2002, 32, 1277-1289. [CrossRef]

31. Carette, J.; Staquet, S. Monitoring and modelling the early age and hardening behaviour of eco-concrete through continuous non-destructive measurements: Part I. Hydration and apparent activation energy. Cem. Concr. Compos. 2016, 73, 10-18. [CrossRef]

32. Piccolo, A.; Lecieux, Y.; Delepine-Lesoille, S.; Leduc, D. Non-invasive tunnel convergence measurement based on distributed optical fiber strain sensing. Smart Mater. Struct. 2019, 28, 045008. [CrossRef] 
33. Turcry, P.; Loukili, A.; Barcelo, L.; Casabonne, J.M. Can the maturity concept be used to separate the autogenous shrinkage and thermal deformation of a cement paste at early age? Cem. Concr. Res. 2002, 32, 1443-1450. [CrossRef]

34. Kamen, A.; Denarié, E.; Sadouki, H.; Brühwiler, E. Evaluation of UHPFRC activation energy using empirical models. Mater. Struct. 2009, 42, 527-537. [CrossRef]

35. Yikici, T.A.; Chen, H.L.R. Use of maturity method to estimate compressive strength of mass concrete. Const. Build. Mater. 2015, 95, 802-812. [CrossRef]

36. Neville, A. Properties of Concrete, 5th ed.; Prentice Hall: Englewood Cliffs, NJ, USA, 2011.

37. American Concrete Institute. Cold Weather Concreting-ACI 306R; Technical Report ACI 306R; American Concrete Institute: Detroit, MI, USA, 2002.

38. American Concrete Institute. Standard Specification for Cold Weather Concreting-ACI 306; Technical Report ACI 306; American Concrete Institute: Detroit, MI, USA, 2002.

39. Lothenbach, B. Thermodynamic equilibrium calculations in cementitious systems. Mater. Struct. 2010, 43, 1413-1433. [CrossRef]

40. Collepardi, M. A state-of-the-art review on delayed ettringite attack on concrete. Cem. Concr. Compos. 2003, 25, 401-407. [CrossRef]

41. Bamforth, P.B. Early-Age Thermal Crack Control in Concrete; CIRIA: London, UK, 2007.

42. Harmathy, T. Effect of Moisture on the Fire Endurance of Building Elements. Moisture Mater. Relat. Fire Tests 1965. [CrossRef]

43. Baroghel-Bouny, V. Caractérisation Microstructurale et Hydrique des Pâtes de Ciment et des Bétons Ordinaires et à Très Hautes Performances. Ph.D. Thesis, Ecole Nationale des Ponts et Chaussées, Paris, France, 1994.

44. Powers, T.C. A discussion of cement hydration in relation to the curing of concrete. Highw. Res. Board Proc. 1948, 27, 178-188.

45. Samouh, H.; Rozière, E.; Loukili, A. The differential drying shrinkage effect on the concrete surface damage: Experimental and numerical study. Cem. Concr. Res. 2017, 102, 212-224. [CrossRef]

46. Lura, P. Autogenous Deformation and Internal Curing of Concrete; Delft University Press: Delft, The Netherlands, 2003.

47. Sellevold, E.; Bj øntegaard, O. Driving forces to cracking in hardening concrete: Thermal and autogenous deformations. In Proceedings of the 2nd International Symposium on Advances in Concrete through Science and Engineering, Quebec City, QC, Canada, 11-13 September 2006.

48. Darquennes, A.; Staquet, S.; Delplancke-Ogletree, M.P.; Espion, B. Effect of autogenous deformation on the cracking risk of slag cement concretes. Cem. Concr. Compos. 2011, 33, 368-379. [CrossRef]

49. Darquennes, A.; Rozière, E.; Khokhar, M.I.A.; Turcry, P.; Loukili, A.; Grondin, F. Long-term deformations and cracking risk of concrete with high content of mineral additions. Mater. Struct. 2012, 45, 1705-1716. [CrossRef]

50. Whittington, H.W.; McCarter, J.; Forde, M.C. The conduction of electricity through concrete. Mag. Concr. Res. 1981, 33, 48-60. [CrossRef]

51. Brameshuber, W.; Dauberschmidt, C.; Schröder, P.; Raupach, M. Non-Destructive Determination of the Water Content in the Concrete Cover Using the Multi-Ring-Electrode; Technical Report RWTH-CONV-006116; DGZfP: Potsdam, Germany, 2003.

52. Burlion, N.; Bourgeois, F.; Shao, J.F. Effects of desiccation on mechanical behaviour of concrete. Cem. Concr. Compos. 2005, 27, 367-379. [CrossRef]

53. McCarter, W.J.; Chrisp, T.M.; Starrs, G.; Basheer, P.A.M.; Blewett, J. Field monitoring of electrical conductivity of cover-zone concrete. Cem. Concr. Compos. 2005, 27, 809-817. [CrossRef]

54. Liu, Z.; Zhang, Y.; Jiang, Q. Continuous tracking of the relationship between resistivity and pore structure of cement pastes. Const. Build. Mater. 2014, 53, 26-31. [CrossRef]

55. Andrade, C.; Castellote, M.; d'Andrea, R. Chloride aging factor of concrete measured by means of resistivity. In Proceedings of the Proceedings of the XII-International Conference on Durability of Building Materials and Components, Porto, Portugal, 12-15 April 2011.

(C) 2019 by the authors. Licensee MDPI, Basel, Switzerland. This article is an open access article distributed under the terms and conditions of the Creative Commons Attribution (CC BY) license (http:/ / creativecommons.org/licenses/by/4.0/). 